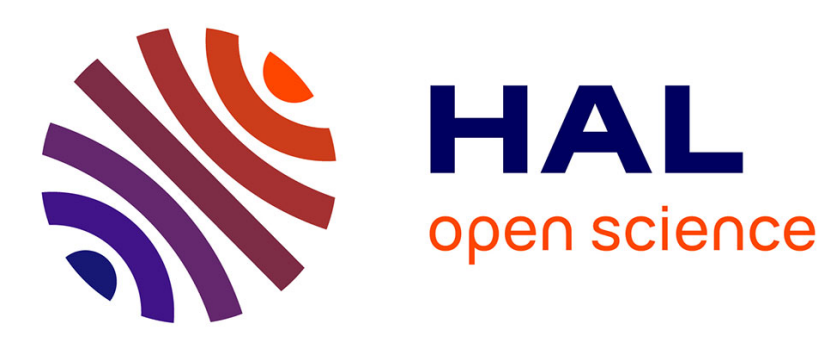

\title{
À la recherche du politique dans les villes Patrick Le Galès
}

\section{To cite this version:}

Patrick Le Galès. À la recherche du politique dans les villes. Raisons politiques, 2020, 2020/3 (79), pp.11 - 40. 10.3917/rai.079.0011 . hal-03053983

\section{HAL Id: hal-03053983 \\ https://hal-sciencespo.archives-ouvertes.fr/hal-03053983}

Submitted on 20 Dec 2021

HAL is a multi-disciplinary open access archive for the deposit and dissemination of scientific research documents, whether they are published or not. The documents may come from teaching and research institutions in France or abroad, or from public or private research centers.
L'archive ouverte pluridisciplinaire HAL, est destinée au dépôt et à la diffusion de documents scientifiques de niveau recherche, publiés ou non, émanant des établissements d'enseignement et de recherche français ou étrangers, des laboratoires publics ou privés. 


\title{
À la recherche du politique dans les villes
}

\author{
Patrick Le Galès
}

La question urbaine interroge depuis longtemps le politique et la démocratie ${ }^{1}$. A priori la question est assez consensuelle comme dans cette formulation de Marcel Roncayolo : « La présence du fait politique est partout dans la ville : la ville exerce ses fonctions politiques ou administratives à l'égard d'un territoire plus ou moins vaste : elle participe à l'encadrement territorial. Elle gère d'autre part ses propres affaires. Mais la ville, lieu de centralité, est également site privilégié de l'expression, de la diffusion des idées, de la lutte aussi. Capitale, elle organise les dominations comme elle couve les révolutions ${ }^{2}$. » En effet, les villes, les métropoles ont été le creuset de formes politiques ensuite nationalisées, ou parfois de formes originales d'ordre politique, plus ou moins stable. Ce sont des espaces politiques à la fois encastrés au sein des États et reliés aux autres villes par des myriades d'échanges, de circulations de biens et de personnes, et de réseaux. Les villes, les métropoles sont ici définies en termes d'accumulation et de concentration (densité) d'individus, de groupes, de bâtiments, d'infrastructures, de relations sociales (formelles ou informelles), de représentations, d'imaginaires, d'organisations, d'institutions et de projets politiques, « lieux de concentration d'un environnement bâti qui du sens où les gens vivent et interagissent, faisceaux délimités de processus sociaux ${ }^{3} \gg$ multiplicateur d'échanges sur fond d'inégalités. Les villes et les régions urbaines sont également des espaces et des structures politiques qui sont plus ou moins régies et régulées par des politiques, des marchés, des arrangements informels, des élites politiques, des entreprises, des Organisations non gouvernementales, des groupes communautaires, des familles, des institutions, des mouvements sociaux, des fonctionnaires, des églises ou des gangs et des mafias.

Premièrement, l'article a pour objet une synthèse des débats interdisciplinaires sur le politique et l'urbain. Si la question urbaine est jusqu'à présent relativement marginale dans la théorie politique et assez peu présente dans la science politique, d'autre disciplines, notamment la sociologie, la géographie, l'anthropologie ont proposé des contributions à ce

\footnotetext{
${ }^{1}$ Moses Immanuel Finley, « The Ancient City: From Fustel de Coulanges to Max Weber and Beyond », Comparative Studies in Society and History, vol. 19, n 3, 1977, p. 305-327. Peter Clark (dir.), The Oxford Handbook of Cities in World History, Oxford, Oxford University Press, 2013. Patrick Le Galès, « The Political Sociology of Cities and Urbanisation Processes: Social Movements, Inequalities and Governance » In, Susan .Hall, Ricky .Burdett (dir),. The Sage Handbook of the 21st Century City, London: Sage,p. 215-235,2017 ${ }^{2}$ Marcel Roncayolo, La ville et ses territoires, Paris, Gallimard, 1997, p. 3

${ }^{3}$ Göran Therborn, 2017 Cities of power: the urban, the national, the popular, the global. Verso Books, p.15.
} 
sujet. Pourtant, la théorie politique et la philosophie politique se sont peu intéressées aux questions urbaines, malgré l'héritage grec ou la réflexion sur le républicanisme des cités italiennes ${ }^{4}$. Pour Jonathan Matthew Hoye, il faut y voir le succès de longue durée de Hobbes et du Léviathan qui a contribué à marginaliser le républicanisme urbain. Sa conception de l'État, classiquement présentée comme une remise en cause de la souveraineté populaire ${ }^{5}$, est aussi une attaque contre le républicanisme urbain et l'autonomie locale. Pour Daniel Weinstock la question urbaine est la grande absente de la philosophie politique ${ }^{6}$ :

À quelques rares exceptions près, la ville n'existe pas aux yeux des philosophes politiques contemporains, ou à tout le moins ne représente-t-elle pas un phénomène digne de figurer aux côtés de l'État-nation comme objet méritant une étude normative approfondie?

Le parti pris de la synthèse et de la présentation de débats est limité dans le cadre de cet article à l'Europe et à l'Amérique du Nord. La première partie permet de caractériser certains traits du politique à l'échelle urbaine en termes de pluralisme, de libéralisme d'une part, de contestation, violence et police d'autre part. Des problèmes d'action collective sont toujours posés pour créer des infrastructures, des équipements et des règles pour l'utilisation du sol ou des biens publics pour régler des conflits ${ }^{8}$. Dans ces conditions, la conception du politique comme tentative d'organisation d'un ordre, « un processus d'accommodation incertain de gestion des contradictions sociales et de réduction des conflits sociaux ${ }^{9} » s^{\prime}$ applique bien aux villes.

Deuxièmement, cet article est une contribution à la compréhension du politique dans les sociétés contemporaines plus ou moins mondialisées envisagé à partir des villes et non de l'État, en faisant l'hypothèse que les processus d'urbanisation et de mondialisation contemporains ouvrent des possibilités de différenciation du politique et des régulations politiques à l'échelle soit transnationale soit infranationale ${ }^{10}$. Cette urbanisation planétaire remet en cause à l'inverse le politique identifié à un lieu et amène à un renouvellement des questions d'inégalités, de justice, de démocratie, de citoyenneté : les individus (migrants par

\footnotetext{
${ }^{4}$ Jonathan Matthew Hoye, « Leviathan against the City Commonwealth », The History of Political Thought, à paraître, 2020.

${ }^{5}$ Quentin Skinner, « A Genealogy of the Modern State », Proceedings of the British Academy, vol. 162, janvier 2009, p. 325-370.

${ }^{6}$ Daniel M. Weinstock, « Pour une philosophie politique de la ville », Rue Descartes, vol. 1, no 63, 2009, p. 6371.

${ }^{7}$ Rainer Bauböck, « Cities vs States: Should Urban Citizenship be Emancipated from Nationality? », in Rainer Bauböck et Liav Orgad (dir.), Cities vs States: Should Urban Citizenship be Emancipated from Nationality?, EUI working paper series, Robert Schuman Centre for Advanced Studies, European University Institute, 2020, p. 1-6, disponible en ligne (https://cadmus.eui.eu/bitstream/handle/1814/66369/RSCAS_2020_16.pdf).

${ }^{8}$ Jane Jacobs, Death and Life of Great American Cities, New York, Random House, $19 \overline{6} 1$.

${ }^{9}$ Jacques Commaille et Bruno Jobert, Les métamorphoses de la régulation politique, Paris, LGDJ, 1998.

${ }^{10}$ James N. Rosenau, Turbulence in World Politics: A Theory of Change and Continuity, Princeton, Princeton University Press, 2018.
} 
exemple) peuvent avoir des droits et des devoirs ainsi que des accès à des services différents selon les territoires au sein d'un même État. Les emboîtements entre niveaux de gouvernements ouvrent des contradictions et des possibilités pour les citoyens.

Troisièmement, et de manière là aussi différenciée de l'État, l'accumulation d'investissements matériels dans les villes en fait des concentrations originales de technologies qui peuvent s'analyser avec la sociologie de sciences comme des assemblages des systèmes sociotechniques où le politique se comprend en termes pragmatiques d'interactions quotidiennes.

L'article est donc organisé afin à la fois de caractériser le politique dans les villes- et de présenter les principaux débats dans la littérature, tout en proposant deux hypothèses en termes de différenciation entre politique des villes et politique des États.

\section{Politique et ville : pluralisme, libéralisme, violence, contestation, domination}

Classiquement, la question du politique et de l'urbain est pensée à partir des villes avant l'État-nation, par exemple la poleis grecque - où l'ensemble des citoyens participent aux décisions et aux travaux communs -, la corporation, la municipalité ou la cité républicaine puis, progressivement, comme gouvernement local ou municipalité au sein de l'État-nation. Dans Le pain et le cirque ${ }^{11}$, Paul Veyne décrit à Rome la concurrence des groupes interdépendants qui caractérise le pluralisme politique urbain, mais aussi l'importance des biens publics dans la ville, des services collectifs, de la fiscalité, de l'oligarchie républicaine qui remet en cause la souveraineté de fait des citoyens. Des poleis grecques aux villes chinoises intégrées par la bureaucratie de l'empire, des villes sophistiquées des mayas, des incas ou des aztèques aux concentrations urbaines au bord de l'Indus, de Rome aux villes africaines, des villes européennes médiévales aux métropoles américaines puis aux mégapoles asiatiques, des conceptions, des imaginaires, des pratiques, des instruments de la politique ont été élaborées dans ces villes plus au moins délimitées, plus ou moins autonomes, plus ou moins organisées par des régulations politiques. Historiquement, la question urbaine contribue à conceptualiser les limites de la régulation politique et à penser le politique au-delà de l'État, notamment à partir de questions de la vie quotidienne, de droits (des pauvres ou des migrants), de citoyenneté, d'accès à des services, des biens publics, des logements ${ }^{12}$. Cependant, en Europe, les villes bourgeoises et commerçantes furent progressivement intégrées au sein des États au fil des siècles, rapidement chez les Britanniques, tardivement en

\footnotetext{
${ }^{11}$ Paul Veyne, Le pain et le cirque : sociologie historique d'un pluralisme politique, Paris Seuil, 1976.

${ }^{12}$ Sur les questions de citoyenneté et la critique du modèle wébérien, voir notamment Engin F. Isin, Being Political. Genealogies of Citizenship, Minneapolis, University of Minnesota Press, 2002.
} 
Italie en Allemagne ${ }^{13}$. Capitalisme, guerres et révolutions contribuèrent à la formation de l'État-nation européen moderne, à la nationalisation de la politique ainsi qu'à l'effacement des villes (au sens de Weber) comme entités politiques autonomes ${ }^{14}$. La formation des villes, leur développement, les processus d'urbanisation ont le plus souvent résulté de la tension entre des dynamiques économiques commerciales, industrielles, financières, de la production, de la consommation, du marché du travail, des migrations, de la circulation, de la propriété, de l'exploitation d'une part, et d'autre part des logiques politiques de contrôle, de sécurité, de défense, d'exercice de l'autorité politique, de citoyenneté, de religion, d'inégalités, de contestations, de démocratie, de planification, de gouvernance, de production de services et d'équipements. Les villes sont en relation avec d'autres villes via de multiples réseaux, mais aussi avec d'autres organisations politiques, de l'empire à maintes autres formes plus ou moins baroques qui sont apparues au cours de l'histoire, d'où des questions de différenciation, d'autonomie, de coopération, de compétition. Ceci soulève aussi une question de définition.

De cette littérature historique émergent plusieurs points clé pour analyser le politique dans les villes. D'abord, elles se caractérisent par des populations diverses, et donc des inégalités, des migrations des croyances, des cultures, des modes de vie différents. Le conflit est central dans les villes pour répondre à des questions classiques : Qui habite au centre ? Qui est taxé ? Qui a accès à des services ? Qui décide de l'utilisation du foncier ? Qui est exclu ? Qui gouverne ? Le politique dans la ville est une question de conflit, de violence qui conduit à des modes de résolution de ces conflits, à de l'ordre et à de la police. La ville est une structure politique pluraliste plutôt libérale : des groupes sont en concurrence, des formes d'autorité sont aussi en concurrence, des choix individuels et collectifs s'exercent. Georg Simmel puis à sa suite Richard Sennett, Iris Marion Young ou Engin F. Isin, ont beaucoup insisté sur ces relations à l'étranger ou aux individus et groupes différents qui caractérisent l'urbanité ${ }^{15}$ et qui différencient le politique de l'urbain de la communauté locale.

\footnotetext{
${ }^{13}$ Jean-Luc Pinol (dir.), Histoire de l'Europe Urbaine, Paris, Seuil, 2003 ; Charles Tilly et Wim P. Blockmans, Cities and the Rise of States in Europe, A.D. 1000-1800, Boulder, Westview Press, 1994.

${ }^{14}$ Maarten Prak, Citizens without Nations: Urban Citizenship in Europe and the World, c. 1000-1789, Cambridge, Cambridge University Press, 2018. Charles Tilly, Contrainte et capital dans la formation de l'Europe 990-1990, trad. fr. Denis-Armand Canal, Paris, Éditions Aubier, 1992. Patrick. .Le retour des villes européennes. Paris, Presses de Sciences Po 2éme édition, 2011.

${ }^{15}$ Georg Simmel, Les grandes villes et la vie de l'esprit, trad. fr. Jean-Louis Vieillard-Baron et Frédéric Joly, Paris, Éditions Payot, 2013. Richard Sennett, The Conscience of the Eye. The Design and Social Life of Cities, Londres, W. W. Norton\&Company, 1990. Richard Sennett, « The Spaces of Democracy », in Robert A. Beauregard et Sophie Body-Gendrot, (dir.), The Urban Moment. Cosmopolitan Essays on the Late-20th-Century City, Thousand Oaks, Sage, 1999 ; Iris Marion Young, Justice and the Politics of Difference, Princeton, Princeton University Press, 1990.
} 
Urban politics dans le cadre étatique

Compte tenu de l'absence de frontière, et en comparaison avec l'État-nation, ces villes ou métropoles ne paraissent pas pertinentes pour penser les questions de justice, de démocratie, de citoyenneté, de solidarité. Pour Daniel Weinstock, et plus encore pour le théoricien canadien Warren Magnusson ${ }^{16}$, la théorie politique reste trop marquée par le cadre étatique alors que la recherche empirique ne cesse de remettre en cause la conception du politique exclusivement pensée dans le cadre de l’État.

Ainsi au sein de la science politique états-unienne, la question politique urbaine a été formalisée en termes d'un sous-domaine un peu étroit, -urban politics, qui a pris pour objet la construction des politiques urbaines dans le cadre étatique. Dans les années 1960, cette littérature a connu son heure de gloire avec les apports des grands auteurs pluralistes (Edward C. Banfield, Robert A. Dahl, Nelson W. Polsby ou Theodore J. Lowi), posant les questions classiques de compétition politique, d'inégalités de ressources politiques et économique, des élites locales, du poids des entreprises et des promoteurs ou de la démocratie locale notamment en lien avec la question ethnique ${ }^{17}$. Historiquement, les machines politiques américaines ont ainsi été essentielles pour intégrer les migrants ${ }^{18}$. Progressivement, cette tradition a été marginalisée au sein de la science politique états-unienne, mais elle a continué d'interroger la gouvernance des villes américaines en particulier sur le rôle des élites et des coalitions plus ou moins démocratiques associant acteurs publics et acteurs privés aux ressources complémentaires (dans la tradition du « qui gouverne »? de Robert A. Dahl) en termes de « régimes politiques urbains », de « coalitions de croissance », d'autonomie des gouvernements urbains à l'égard des États ou des intérêts économiques ou de leadership des maires. D'autres travaux ont perpétué l'analyse des politiques sectorielles urbaines dans les domaines des transports, du logement, de l'urbanisme, des infrastructures, de la prise en charge des pauvres, du développement économique, des politiques culturelles, et surtout de la police et du développement économique ${ }^{19}$. Une partie des outils classiques de la science politique ont été mobilisés pour analyser les comportements politiques urbains. Dans toute cette littérature, les villes ont été analysées comme un élément du système politique états-

\footnotetext{
${ }^{16}$ Warren Magnusson, Politics of Urbanism: Seeing Like a City, Abingdon/New York, Routledge, 2013 [2011]. ${ }^{17}$ Voir l'ouvrage fondateur Robert. A. Dahl, Who Governs? Democracy and Power in an American City, New Haven/Londres, Yale University Press, 1961.

${ }^{18}$ François Bonnet, « Les machines politiques aux États-Unis. Clientélisme et immigration entre 1870 et 1950 », Politix, vol. 4, n 92, 2010, p. 7-29.

${ }^{19}$ Karen Mossberger, Susan E. Clarke et Peter John (dir.), The Oxford Handbook of Urban Politics, Oxford, Oxford University Press, 2015 ; Joshua Sapotichne, Bryan D. Jones, Michelle Wolfe, « Is Urban Politics a Black Hole? Analyzing the Boundary between Political Science and Urban Politics », Urban Affairs Review, vol. 43, $\mathrm{n}^{\mathrm{o}} 1,2007$, p. $76-106$.
} 
unien, mais la crise urbaine et industrielle des métropoles du nord-est des années 1970 s'est accompagnée du déclin des recherches urbaines dans la science politique. En Europe, les Britanniques ont importé de nombreux travaux américains et ont organisé ce domaine avec les mêmes questions et concepts (régimes urbains, relations ethniques, leadership, etc.) combinées à des réflexions sur le gouvernement local et la démocratie locale héritées de Stuart Mill20.

Par ailleurs, à partir de la fin des années 1960, la vague marxiste renouvelle profondément la recherche et fait de la « question urbaine » un point d'entrée alternatif pour le politique. L'urbanisation en est le concept central, alors que la ville est dissoute dans ce processus d'urbanisation et perd toute pertinence théorique ou analytique. L'urbain devient crucial pour le politique défini en termes de conflits et de lutte des classes ${ }^{21}$. Les auteurs marxistes nient largement l'autonomie du politique, la capacité d'action collective des acteurs urbains ou les effets possibles de transformation de l'action publique sauf dans l'intérêt des dominants capitalistes ${ }^{22}$. Pour le plus important d'entre eux, le géographe David Harvey, les villes ne peuvent en aucun cas contribuer à une explication de phénomènes sociaux ou politiques puisqu'elles résultent de processus d'urbanisation intrinsèquement liés à un type de capitalisme (marchand, industriel, fordiste ou financier, néolibéral et globalisé aujourd'hui) ${ }^{23}$. Pour ces auteurs, les politiques urbaines sont toujours une ruse du capital qui produit de la pauvreté, de l'exclusion et de la gentrification. Le seul politique qui vaille a pour objet la lutte des classes et la révolution.

Progressivement cependant, certains auteurs vont développer des analyses du pouvoir local reprenant une partie de l'analyse marxiste tout en mettant en évidence une certaine autonomie du politique et du pouvoir local ainsi que le rôle du capitalisme d'État ${ }^{24}$.

\section{La contestation}

Ce qu'on a appelé « la nouvelle sociologie urbaine » - c'est-à-dire la sociologie urbaine marxiste et néomarxiste novatrice des années 1970 - a alors mis au cœur de l'analyse la

\footnotetext{
${ }^{20}$ Jonathan Davies et David Imbroscio (dir.), Theories of Urban Politics, Londres, Sage, 2009 ; Desmond King et Gerry Stoker (dir.), Rethinking Local Democracy, Basinstoke, Macmillan, 2014.

${ }^{21}$ Manuel Castells, La question urbaine, Paris, François Maspero, 1972.

${ }^{22}$ Ira Katznelson, Marxism and the City, Oxford, Clarendon Press, 1993 [Oxford University Press, 1992].

${ }^{23}$ David Harvey, Consciousness and the Urban Experience: Studies in the History and Theory of Capitalist Urbanization, 1, Baltimore, Johns Hopkins University Press, 1985 ; David Harvey, The Urbanization of Capital: Studies in the History and Theory of Capitalist Urbanization 2, Oxford, Blackwell, 1987.

${ }^{24}$ En France, c'est l'influence de Poulantzas qui est marquante, $c f$. Sylvie Biarez et al. (dir.), Institution communale et pouvoir politique, le cas de Roanne, Paris/La Haye, Mouton, 1973. Pour la Grande-Bretagne, Patrick Dunleavy, Urban Political Analysis: The Politics of Collective Consumption, Londres, Macmillan, 1980. Peter Saunders, Urban Politics: A Sociological Interpretation, Londres, Routledge, 2006.
} 
contestation du système capitalisme et, dans sa version urbaine, les mouvements sociaux dans la structuration de la ville, la dynamique des relations sociales et les changements politiques urbains. Les mouvements sociaux urbains ont été définis comme des mobilisations collectives (distinctes des partis politiques) des populations urbaines, visant à changer les politiques, s'opposant à des opérations d'aménagement et des projets urbains, défendant leurs intérêts (leur quartier, leur logement), impliquant des actions et des répertoires très divers non institutionnalisés dans les partis politiques, mobilisant contre les inégalités ${ }^{25}$. La dimension urbaine signifie, outre l'échelle, que ces mouvements concernent surtout 1) les questions de consommation collective, d'inégalités et de services publics, 2) les questions de qualité de vie dans le quartier et l'opposition aux changements physiques prévus, et 3) les demandes de transparence et de participation démocratique au gouvernement urbain, ainsi que la contestation des représentants élus et des partis ${ }^{26}$.

Cette dimension reste essentielle pour la question politique urbaine. En effet, les mouvements sociaux urbains sont plus importants que jamais, du mouvement Occupy à la mobilisation contre la privatisation de l'autoroute à Santiago du Chili, l'exclusion des établissements informels pour faire place aux logements de la classe moyenne ou aux centres commerciaux à Mumbai, Johannesburg ou Rio, pour mobiliser pour ou contre l'accueil des réfugiés dans les villes européennes, pour protester contre la construction d'un nouvel aéroport à Mexico, contre l'incurie de la gestion des déchets à Beyrouth, contre les coupures d'électricité, contre la hausse du prix du pain à Casablanca, contre la concentration des sites d'Airbnb dans les vieux quartiers de Barcelone et de Berlin, raréfiant la disponibilité de logements pour la population locale ${ }^{27}$. La vie démocratique de nombreuses métropoles repose sur des conflits et des mobilisations autour de la mixité des quartiers, de l'utilisation de l'espace public par les toxicomanes, des conflits interethniques, religieux, xénophobes et de classe, y compris les groupes d'intérêts les plus progressistes et les plus conservateurs, et de la

\footnotetext{
${ }^{25}$ John Walton, « Urban Conflict and Social Movements in Poor Countries: Theory and Evidence of Collective Action », International Journal of Urban and Regional Research, vol. 22, nº 3, 1998, p. 460-481; Chris Pickvance, « From Urban Social Movements to Urban Movements: A Review and Introduction to a Symposium on Urban Movements », International Journal of Urban and Regional Research, vol. 27, n 1, 2003, p. 102-109. ${ }^{26}$ Massimiliano Andretta, Gianni Piazza, Anna Subirats, « Urban Dynamics and Social Movements », in Donatella Della Porta et Mario Diani (dir.), The Oxford Handbook of Social Movements, New York, Oxford University Press, 2015, p. 200-218 ; David Harvey, Rebel Cities: From the Right to the City to the Urban Revolution, Londres/New York,Verso, 2012 ; Claire Colomb et Johannes Novy, Protest and Resistance in the Tourist City, Londres/New York, Routledge, 2016 ; Eric Verdeil, « The Energy of Revolts in Arab cities: The case of Jordan and Tunisia », Built Environment, vol. 40, nº 1, 2014, p. 128-139.

${ }^{27}$ Voir par exemple Gianpaolo Baiocchi, Militants and Citizens, Stanford, Stanford University Press, 2005 ; Thomas Aguilera, Gouverner les illégalismes urbains. Les politiques publiques face aux squats et aux bidonvilles dans les régions de Paris et de Madrid, Paris, Dalloz, 2017 ; ; Walter. J Nicholls et Justus Uitermark, Cities and Social Movements: Immigrant Rights Activism in the US, France, and the Netherlands, 1970-2015, Chichester/Malden, John Wiley \& Sons, 2016.
} 
mobilisation. Les dynamiques classiques comprennent la lutte pour les biens collectifs, contre les grands projets d'urbanisme et la quête de reconnaissance publique via des procédures contentieuses. Les formes actuelles de contestation urbaine sont affectées par des problèmes de propriété privée, d'ethnicité ou de lutte contre la police, d'expulsion des quartiers pauvres, de modes de vie et d'individualisation, de densité, de migrations alternantes et d'utilisation des espaces et des transports publics, d'entre-soi des classes moyennes supérieures, d'inégalités territoriales et de revendications de péréquation, de localisation des infrastructures polluantes, de revendications d'accès aux services, de l'eau à l'éducation.

\section{Au sein et au delà de l'État, Seeing like a city : désencastrement et gouvernance}

Des villes leaders et cibles

Alors que pendant les années 1970 la crise industrielle et les idées post-68 avaient fait de la « fin des villes » un thème de prédilection, depuis le début des 1980, une vague puissante d'urbanisation liée aux processus de globalisation a modifié les équilibres territoriaux au profit des villes, des métropoles des régions urbaines ${ }^{28}$. La montée en puissance des grandes métropoles et des villes moyennes sur tous les continents ainsi que la diversité des processus d'urbanisation contribuent, avec bien d'autres, à remettre en question la compréhension classique du politique comme étant historiquement lié à l'institutionnalisation de l'État. Le simple changement d'échelles des mégapoles et leur dynamique économique brouille les échelles de comparaison et complexifie les enchevêtrements au sein des États. Steven Graham montre par exemple comment les villes sont devenues essentielles dans les stratégies militaires et comment leur destruction est devenue prioritaire dans les guerres ${ }^{29}$.

En effet, le $21^{\mathrm{e}}$ siècle connaît une expansion urbaine sans précédent en termes de taille des villes, de vitesse d'urbanisation, d'étendue géographique, de diversité des formes sociales et bâties, de modes d'organisation et de gouvernance des villes. On attend 800 millions de personnes supplémentaires dans les métropoles dans la prochaine décennie, une trentaine de métropoles vont dépasser les 15 millions d'habitants. Dans certains cas, des méga-régions urbaines d'une taille gigantesque voient le jour, comme Jin Ji Ji (Beijing et ses environs) prévue pour 100 millions d'habitants considérée comme la future capitale du monde par le gouvernement chinois, mais aussi Delhi-Gurgaon-Gazhiabad en Inde, Shangai ou Tokyo-

\footnotetext{
${ }^{28}$ Pierre Veltz, Mondialisation, villes et territoires. L'économie d'archipel, Paris, PUF, 1996 ; Michael Storper, Keys to the City: How Economics, Institutions, Social Interaction, and Politics Shape Development, Princeton, Princeton University Press, 2013.

${ }^{29}$ Steven Graham, Cities under Siege: The New Military Urbanism, Londres/New York,Verso, 2011.
} 
Osaka, qui comptent toutes déjà environ 30 millions d'habitants. À l'échelle de 2100, Lagos, Dhakka, Dar Es Salaam voire Kinshasa pourraient compter 70 à 80 millions d'habitants ${ }^{30}$. Cependant, le gigantisme n'est qu'une partie de l'histoire. Une grande partie de la croissance urbaine mondiale se produit dans des villes de taille moyenne (entendues comme des zones métropolitaines de 500- 000 à 5 millions d'habitants), en Europe, au Canada, dans certaines parties de l'Afrique, en Inde et en Chine. Les 50 plus grandes villes du monde concentrent $7 \%$ de la population mondiale, mais elles représentent $40 \%$ de la production économique mondiale, - souvent le moteur de l'économie nationale - et plus d'un quart de l'empreinte carbone globale ${ }^{31}$, surtout les métropoles les plus riches. Les villes et les métropoles sont des lieux d'innovation culturelle, de développement économique, d'accueil de migrants, de mobilité sociale, de diversité, de politiques publiques. Elle sont aussi des lieux de violence parfois extrême, d'insécurité, de pauvreté, d'exclusion, d'oligarchies, de révoltes, de désordre politique, de gangs, de déclin et d'informalité ${ }^{32}$.

Contestées par les mouvements populistes et nationalistes, fragilisées par la crise climatique et les risques d'inondation, de sécheresse, de manque de nourriture ou d'énergie, ou par les épidémies, elles font preuve de fragilité. Les inégalités s'y déploient de manière massive et les questions d'ordre politique, de contestation, de conflits, d'illégalisme, de cibles militaires ou terroristes sont majeures. De nouvelles façons de construire des villes existent parallèlement à une crise mondiale du logement et à l'expansion continue des bidonvilles, des sans-abri et de l'enfermement des plus riches dans des ghettos ${ }^{33}$. Les hausses massives des prix des logements à Tokyo, San Francisco, Shanghai, Vancouver ou Luanda, New York, Londres et Paris produisent un nouveau groupe de rentiers. Les villes et les grandes métropoles ne se contentent pas de refléter les inégalités, elles deviennent des moteurs majeurs produisant et réduisant des inégalités via la ségrégation des élites, les impôts, les politiques redistributives ou l'accumulation du patrimoine hérité dû à la spectaculaire hausse des prix du logement ${ }^{34}$.

\footnotetext{
${ }^{30}$ Estimation du Global Cities Centre, University of Toronto (https://www.globalcitiesinstitute.org/).

${ }^{31}$ Chiffres du Earth Institute de Columbia (https://www.earth.columbia.edu/).

${ }^{32}$ Gabriel Feltran, The Entangled City: Crime as Urban Fabric in São Paulo, Manchester, Manchester University Press, 2020 ; Suzanne M. Hall, « Mooring “super-diversity” to a brutal migration milieu », Ethnic and Racial Studies, vol. 40, nº 9, 2017, p. 1562-1573.

${ }^{33}$ Mike Davis, Planet of Slums, Londres /New York, Verso, 2006 ; Robert Neuwirth, Shadow Cities: A Billion Squatters. A New Urban World, Londres, Routledge, 2005; Ray Forrest, Sin Yee Koh, Bart Wissink (dir.), Cities and the Super-Rich: Real Estate, Elite Practices and Urban Political Economies, New York, Palgrave Macmillan, 2017.

${ }^{34}$ Patrick Le Galès, P., Paul Pierson, "Superstar Cities" \& the Generation of Durable Inequality. Dcedalus, 148(3), 46-72, 2019. Robert Beauregard, Cities in the urban age: A dissent. Chicago, University of Chicago Press 2018
} 
La ségrégation et la reproduction des élites est un phénomène durable, qui est constamment mis en œuvre par les pratiques de mobilité. Les populations pauvres sont tenues à l'écart, déplacées, parfois dans des ghettos ou des bidonvilles à la lointaine périphérie.

Seeing like a city?

Afin d'esquisser ce politique du monde des villes et, en paraphrasant James Scott, on peut tenter de formaliser ou de mesurer ce que pourrait vouloir dire la formule Seeing like a city : c'est-à-dire penser les villes autrement que comme des mini États ou un type d'espace gouverné par les logiques de l'État avec de faibles marges de différenciation. Les travaux sur la démocratie locale, le municipalisme ou l'urbanisme ont ainsi nourri des débats sur la dynamique urbaine ou locale du politique ${ }^{35}$. Des chercheurs ont réfléchi aux questions urbaines en remettant en cause les formulations en termes de nationalisation politique. Parallèlement au renouveau de l'intérêt pour les territoires, ils se sont interrogés sur des mondes politiques urbains différenciés, sur des logiques du gouvernement urbain, sur la reterritorialisation politique dans les villes, et ont même tenté d'élaborer une analyse écologique du politique métropolitain ${ }^{36}$. Tout ceci a été peu lié aux débats de théorie politique qui ont laissé de côté la question urbaine. Jonathan Matthew Hoye voit dans les écrits d'Althusius, plus connu pour ses travaux sur le fédéralisme et sa critique de Hobbes, la justification normative pour la formation des villes ainsi qu'une conception dynamique de la politique basée sur l'association, l'autonomie :

Pour Althusius, l'identification des problèmes, des désirs et des objectifs est une pratique communale et communicative. Althusius définit la politique - il utilise le terme symbiotique - comme « l'art d'associer les hommes dans le but d'établir, de cultiver et de conserver la vie sociale entre eux ». Selon Althusius, la politique désigne les flux communicatifs, économiques, sociaux et administratifs de la vie urbaine, mis en ordre par des compromis, des accords et des concessions manifestes nécessaires pour que les citoyens vivent ensemble en paix. La politique n'est donc pas architectonique, elle est une propriété émergente et dynamique de la vie en commun ${ }^{37}$.

À sa manière, Warren Magnusson est l'héritier contemporain de cette perspective. Il a systématiquement tenté de caractériser la ville comme une organisation politique structurée

\footnotetext{
${ }^{35}$ Warren Magnusson, The Search for Political Space, Toronto, University of Toronto Press, 1996 [1991].

${ }^{36}$ Jefferey M. Sellers et al., The Political Ecology of The Metropolis: Metropolitan Sources of Electoral Behaviour in Eleven Countries, Colchester, ECPR press, 2013. Jefferey M. Sellers et al. (dir.), Inequality and Governance in The Metropolis: Place Equality Regimes and Fiscal Choices in Eleven Countries, New York, Palgrave Macmillan, 2016.

${ }^{37} \ll$ For Althusius, identifying problems, desires and goals is a communal and communicative practice. Althusius defines politics- he uses the term symbiotics- as the "art of associating men for the purpose of establishing, cultivating and conserving social life among them. To Althusius, politics denotes the communicative, economic, social and administrative flux of urban live, given order by manifest compromises, agreements, and concessions necessary for citizens to live together in peace. Politics are therefore not architectonic; they are emergent and dynamic properties of communal life". » : Jonathan Matthew Hoye, « Leviathan against the City Commonwealth », art. cité, p. 4 (notre traduction).
} 
par l'absence de souveraineté et le politique de l'urbain comme « une diversité au plus proche, des modèles compliqués de gouvernement et d'autonomie, une multiplicité d'autorités dans des registres différents, l'ajournement sans fin de la souveraineté, l'auto-organisation, et un ordre émergent qui, bien que chaotique, n'est en aucun cas anarchique ${ }^{38} \gg$. Cet auteur insiste, à l'instar de Simmel, sur la relation entre urbanisation et individualisme. Il souligne surtout la capacité des urbains à se gouverner eux-mêmes, à élaborer des règles informelles de vie en commun qui caractérisent la vie urbaine, à résoudre de manière pragmatique des problèmes collectifs, des interactions déstabilisantes. Pour lui, la ville est par nature caractérisée par une forme de libéralisme et de pragmatisme à la Dewey où des expérimentations sont possibles. C'est d'ailleurs pendant le $19^{\mathrm{e}}$ siècle libéral anglais que les auteurs classiques élaborent la doctrine du gouvernement local, du self government, en particulier Stuart Mill.

L'effort de Magnusson consiste à opposer de manière radicale le politique pensé par l'État : autorité, hiérarchie, coercition, stabilité et le politique de l'urbain : pluralité des formes d'autorité, instabilité, relations horizontales, faible capacité du centre, pluralisme, incertitude, transformation continue. Il essaye d'opérer une distinction radicale entre le politique vu du côté de l'État caractérisé selon James Scott par la hiérarchie, la souveraineté, la bureaucratie, les instruments de normalisation, de surveillance et de contrôle, et le politique vue des villes, celui des relations horizontales, de la diversité, des formes de gouvernement et d'autogouvernement, de contrôle de soi au-delà de l'État, non souverain, autonomes, avec un espace public original qui a pris la forme des municipalités dans différentes pays.

Cette opposition peut être pensée dans une dynamique historique. La perspective wébérienne permet d'envisager une dynamique contemporaine de différenciation, d'autonomisation, de désencastrement des métropoles, ou d'une partie d'entre elles.

Dénationalisation et désencastrement : cities are back in town

Dans son analyse de l'âge d'or des villes européennes à la fin de la période médiévale, Max Weber ${ }^{39}$ a souligné la confusion et le chevauchement des pouvoirs entre l'effondrement de l'ordre féodal, la dynamique des villes du premier âge du capitalisme, la mosaïque des autorités politiques, l'Église, la lex mercatoria qui régit les échanges et la lente émergence de

\footnotetext{
${ }^{38}$ « Proximate diversity, complicated patterns of government and self government, a multiplicity of authorities in different registers, the infinite diefferal of sovereignty, self-organisation, and an emergent order, that, though chaotic, is by no means anarchic... » : Warren Magnusson, Politics of Urbanism..., op. cit., p. 109 (Notre traduction).

${ }^{39}$ Max Weber, La ville, trad. fr. Philippe Fritsch, Paris, La Découverte, 1982. Charles Tilly, Contrainte et capital..., op. cit.
} 
l'État. Pour Weber, les bourgeoisies européennes du premier âge du capitalisme marchand devaient défendre, organiser, promouvoir leurs entreprises et leurs villes contre les règles de l'Église, l'aristocratie foncière et la puissance émergente des États territoriaux qui visaient à les taxer et à mobiliser leurs ressources pour leur propre compte. Pour Weber, la fin de la période médiévale et le début de la Renaissance marquent l'âge d'or des villes européennes. L'imbrication des pouvoirs politiques (empires, villes-États, royaumes, duchés, églises-États etc.) est un puissant facteur qui créée des marges de manœuvre pour les élites urbaines, l'organisation collective des villes et l'élaboration de règles et d'institutions pour favoriser les échanges. Aucune autorité politique ne pouvait revendiquer le monopole de l'exercice de l'autorité politique, des droits et des impôts. L'enchevêtrement des échelles et des pouvoirs a été un facteur crucial pour rendre possible l'autonomie politique des villes et leur accumulation de ressources. Progressivement, les États territoriaux se sont institutionnalisés. $\mathrm{Si}$ au sein des villes industrielles victoriennes ${ }^{40}$, les élites de la bourgeoisie commerçante et industrielle organisent le développement économique, construisent des infrastructures, entrent en compétition avec les autres villes et s'occupent à peine des populations ouvrières, l'autonomie du gouvernement local britannique sera progressivement réduite à la portion congrue au cours du $20^{\mathrm{e}}$ siècle. En France, le double effet de la Révolution et de l'industrialisation tardive marginalise assez vite les villes en tant qu'entités politique autonomes. La Commune achève de faire de Paris la ville révolutionnaire du $19^{\mathrm{e}}$ siècle, un risque politique pour l'État qui tient à en limiter l'autonomie. Partout en Europe, pour reprendre l'expression de l'historien Wim Blockmans, les voracious states-auront raison des obstructing cities $^{41}$. À partir de 1870, 1'Europe connaît un siècle de nationalisation sociale, économique et politique, de renforcement du poids de l'État, de «mise en cage » de la société et donc des villes, par la guerre et l'État providence ${ }^{42}$. Au début du $20^{\mathrm{e}}$ siècle en Allemagne, en Angleterre, en France, à Vienne, des mouvements de socialisme municipal visent à inventer des formes de régulation politique originale au service de la classe ouvrière valorisant la participation politique, l'autogestion, les services à la population ouvrière. Ils seront écrasés, marginalisés ou limités dans leur capacité43.

\footnotetext{
${ }^{40}$ Asa Briggs, Victorian Cities. Vol. 2, Oakland, Berkeley, University of California Press, 1993.

${ }^{41}$ Wim Blockmans, Voracious States and Obstructing Cities: An Aspect of State Formation in Preindustrial Europe, Theory and Society, vol. 18, 1989, p. 733-755.

${ }^{42}$ Michael Mann, « Has Globalization Ended the Rise and Rise of the Nation-State? », Review of International Political Economy, vol. 4, n 3, 1997, p. 472-496.

${ }^{43}$ Parmi de nombreuses références : Henry Johns Gibbons, « The Opposition to Municipal Socialism in England », Journal of Political Economy, vol. 9, n 2, 1901, p. 243-259; Aude Chamouard, Une autre histoire du socialisme : les politiques à l'épreuve du terrain (1919-2010), Paris, Éditions du CNRS, 2013 ; Emily
} 
La période contemporaine peut être analysée dans cette perspective wébérienne compte tenu des processus de mondialisation, des formes transnationales de l'autorité privée et publique ${ }^{44}$, de la reconfiguration des États-nations conduisant à une confusion qui découle de l'enchevêtrement des échelles et des gouvernements, de l'augmentation de la mobilité et des échanges ${ }^{45}$. Les réseaux et les relations se sont multipliés et les villes ou les métropoles ne sont pas seulement nationales, mais de plus en plus « des villes dans un monde de villes ${ }^{46}$ », autant que dans un monde d'États, d'organisations transnationales, d'organismes de réglementation privés, de grandes entreprises, d'ONG et de villes de taille moyenne. Lorsqu'aucune autorité politique ne peut revendiquer un monopole (qui n'est jamais pleinement réalisé) à une époque de transformation du capitalisme et d'urbanisation massive de la production et de la consommation de richesses, il faut s'attendre à voir plus d'autonomie politique pour les villes et les métropoles, des formes de différenciation par rapport à l'État et éventuellement le retour de formes de villes-États (Dubaï, Singapour, Panama). Demain, les nouvelles métropoles privatisées imaginées dans différents coins du globe (Amérique du Sud, Gurgaon en Inde), pourraient bien être la conséquence des processus de mondialisation et de la reconfiguration de formes d'autorité politique plus enchevêtrées et moins centrées sur l'État.. La relation entre les grandes métropoles et les États en seraient modifiées : les premières bénéficieraient d'une plus grande autonomie, en étroite coopération, mais aussi en compétition et en conflit avec l'État, tout en étant moins connectées à la société nationale ${ }^{47}$.

Dans cette perspective, aussi immenses, monstrueuses ${ }^{48}$, dynamiques, inégales et dominantes que soient les métropoles, la relation avec l'État reste une variable clé ${ }^{49}$. Le sociologue comparatiste Göran Therborn critique l'illusion des géographes qui se concentrent sur l'étiquetage de divers types de villes « mondiales » ou « globales » les unes par rapport

\footnotetext{
Robinson, « Defining Progressive Politics: Municipal Socialism and Anti-Socialism in Contestation, 1889$1939 »$, Journal of the History of Ideas, vol. 76, n 4, 2015, p. 609-631.

${ }^{44}$ Bertrand Badie, La fin des territoires. Essai sur le désordre international et sur l'utilité sociale du respect, Paris, Fayard, 2014 ; Philipp Genschel et Bernhard Zangl, « State Transformations in OECD Countries », Annual Review of Political Science, vol. 17, 2014, p. 337-354. Miguel Centeno et al. (dir.), States in the Developing World, Cambridge/New York, Cambridge University Press, 2017.); Desmond King, Patrick Le Galès, (dir.), Reconfigurating European states in crisis, Oxford, Oxford University Press, 201

${ }^{45} \mathrm{Du}$ moins A. C. (avant coronavirus). Patrick Le Galès "States in Europe: uncaging societies and the limits to the infrastructural power". Socio-Economic Review, 12(1), 131-152, 2014

${ }^{46}$ Jenny Robinson, «Cities in a World of Cities: The Comparative Gesture », International Journal of Urban And Regional Research, vol. 35, $\mathrm{n}^{\circ}$ 1, 2011, p. 1-23.

${ }^{47}$ Daniel Kübler et Michael A. Pagano, « Urban Politics as Multilevel Analysis » (2012), in Karen Mossberger, Susan E. Clarke et Peter John (dir.), The Oxford Handbook of Urban Politics, op. cit.

${ }^{48}$ Sandrine Lefranc, « Villes-monde, villes monstres? », Raisons politiques, $n^{\circ} 3$, 2004, p. 5-7.

${ }^{49}$ Charles Tilly, «Cities, states, and trust networks: chapter 1 of Cities and States in World History », in Michael Hanagan et Chris Tilly (dir.), Contention and Trust in Cities and States, Dordrecht, Springer, 2011, p. 1-16; Ted Robert Gurr et Desmond S. King, The State and the City, Chicago, The University of Chicago Press, 1987. Jonathan Matthew Hoye, « Leviathan against the City Commonwealth », art. cité.
} 
aux autres, avec une faible compréhension des relations avec l'État ${ }^{50}$. Dans de nombreuses parties du monde, une tendance à la décentralisation conduit à une plus grande autonomie politique des villes et des régions et à une capacité accrue des élites urbaines qui entrent en interaction conflictuelle avec l'État ${ }^{51}$. Ceci a conduit également à une plus grande autonomie politique des villes, à des très fortes augmentations de budget, à l'institutionnalisation des maires et du gouvernement urbain, à la création de coalitions, à des politiques urbaines de grande ampleur dans les domaines classiques de lutte contre la pauvreté ou de constructionrénovation des infrastructures, autant à Lagos et Mexico qu'à São Paolo, New York, Paris, Londres, Milan ou Djakarta ${ }^{52}$. En termes foucaldiens, les villes, ou du moins une partie d'entre elles sont de plus en plus gouvernementalisées ${ }^{53}$.

Les États se reconfigurent et la relation État-métropoles de coopération/conflit contribue à des mobilisations d'habitants, des stratégies, des relations horizontales, des formes d'action collective plus spécifiquement urbaines, mais dont la logique s'inscrit souvent en partie dans l'intégration au sein de l'État, y compris sous forme conflictuelle. Les villes sont un puissant facteur de reconfiguration des États : non seulement leurs populations peuvent être une menace pour l'ordre public, mais par l'accumulation de richesses des élites économiques les élites politiques urbaines ont des ressources pour modifier les priorités de l'État. Pour leur part, ces villes sont aussi soumises aux effets des reconfigurations des États, notamment des processus de dénationalisation de l'autorité politique ${ }^{54}$ qui peuvent amener les élites étatiques à renforcer certaines formes de contrôle ou à prendre leur distance et à se désintéresser des problèmes collectifs de ces villes et de leur citoyen par exemple en termes de logement, de

\footnotetext{
${ }^{50}$ Göran Therborn, Cities of Power: The Urban, the National, the Popular, the Global, Londres/New York, Verso, 2017.

${ }^{51}$ Nirmala Rao, Cities in Transition: Growth, Change and Governance in Six Metropolitan Areas, Londres, Routledge, 2008.; Laurent Fourchard, Trier, exclure et policer: vies urbaines en Afrique du Sud et au Nigeria, Paris, Presses de Sciences Po, 2018. Tulia G. Falleti, Decentralization and Subnational Politics in Latin America, New York, Cambridge University Press, 2010 ; Fulong Wu, « China’s Emergent City-Region Governance: A New Form of State Spatial Selectivity through State-Orchestrated Rescaling », International Journal of Urban and Regional Research, vol. 40, $\mathrm{n}^{\circ}$ 6, 2016, p. 1134-1151; Alison E. Post, « Cities and Politics in the Developing World », Annual Review of Political Science, vol. 21, 2018, p. 115-133.

${ }^{52}$ Michael Jones-Correa (dir.), Governing American Cities: Inter-Ethnic Coalitions, Competition, and Conflict, New York, Russell Sage Foundation, 2001 ; Eduardo Marques, The Politics of the Urban in São Paulo, Oxford, Wiley, à paraître en 2020 ; Alberta Andreotti, Governare Milano nel nuovo millennio, Bologne, Il Mulino, 2019 ; Patrick Le Galès et Vicente Ugalde (dir.), Gobernando la Ciudad de México: Lo que se gobierna y lo que no se gobierna en una gran metrópoli, Mexico, El Colegio de Mexico A. C., 2018 ; Fortunata Piselli, Luigi Burroni, Francesco Ramella, Governare città, Roma, Donzelli, 2012 ; Julie Pollard et Charlotte Halpern, « Les acteurs de marché font-ils la ville ? », EspacesTemps.net, $n^{\circ} 17,2013$.

${ }^{53}$ Pascale Laborier et Pierre Lascoumes, «L'action publique comprise comme gouvernementalisation de l'État », in Sylvain Meyet (dir.), Travailler avec Foucault. Retours sur le politique, Paris, L'Harmattan, 2005, p. 37-60 ; Michel Foucault, Sécurité, Territoire, Population. Cours au Collège de France, 1977-1978, Paris, EHESS/Gallimard/Seuil, 2004.

${ }^{54}$ Stephan Leibfried, Evelyne Huber, Matthew Lange, Jonah D. Levy, Frank Nullmeier, John. D. Stephens (dir.), The Oxford Handbook of Transformations of the State, Oxford, Oxford University Press, 2015.
} 
transport ou de pollution de l'air.

\section{Gouvernance urbaine}

Les villes et les zones métropolitaines apparaissent de plus en plus comme des échelles où se développent les réponses politiques et de gouvernance au désordre, au-delà - et d'une certaine manière en conflit - avec les priorités de leurs États ${ }^{55}$. En effet, les élites des villes et des régions s'efforcent de répondre aux problèmes de la planète ${ }^{56}:$ changement climatique, création d'emplois et développement économique, approvisionnement en eau, interactions entre les différents groupes ethniques et religieux, lutte contre la pauvreté, sécurité et maintien de l'ordre.

Poursuivant la tradition wébérienne, un courant d'analyse a mis la question de la gouvernance et de l'innovation au cœur de la réflexion sur les métropoles, l'urbain et la politique. Beaucoup d'urbanistes considèrent qu'une grande métropole est par principe ingouvernable, beaucoup trop compliquée. Les réseaux, les interdépendances, les mobilités et les circulations sont devenues fondamentales parfois aux dépens des frontières, des institutions, des élus, des citoyens qui travaillent et vivent dans le même territoire. La recherche en histoire, en sociologie ou en économie a montré depuis longtemps que les métropoles sont en partie le résultat de myriades de choix des individus, des ménages, des entreprises, des interactions entre ces différentes composantes. Les économistes ou les tenants de la géographie économique insistent sur la fragmentation de l'exercice de l'autorité politique, la multiplication des agences, les enchevêtrements entre niveaux, les interdépendances massives, des géographies morcelées, des réseaux et des liens horizontaux. Les travaux d'économistes montrent bien les besoins de gouvernance dans la métropole, ne serait-ce que pour résoudre les conflits liés à l'utilisation du sol ou à la gestion des externalités. Cette littérature est assez fataliste et suggère, dans la logique de la théorie des choix publics, que les acteurs expriment leur préférence, créent des institutions partielles et instables, votent « avec leur pied ${ }^{57} »$. Les économistes urbains sont souvent farouchement opposés à la création de gouvernement urbain (qualifié de big government) puisque le politique n'a d'autre légitimité que d'intervenir en cas d'échec du marché. De manière plus sophistiquée, Michael Storper ou Dominique Lorrain ${ }^{58}$ partent de ce point de vue pour

\footnotetext{
${ }^{55}$ Paolo Perulli, The Urban Contract: Community, Governance and Capitalism, Londres, Routledge, 2016.

${ }^{56}$ Julie-Anne Boudreau, Global Urban Politics, Bristol, Polity Press, 2017.

${ }^{57}$ Edward Glaeser, Triumph of the City. How Urban Spaces Make Us Human, Londres, Pan Books, 2012 [Picador, 2011].

${ }^{58}$ Michael Storper, « Governing the Large Metropolis », Territory, Politics, Governance, vol. 2, n 2, 2014, p. 115-134 ; Dominique Lorrain (dir.) Métropoles XXL en pays émergents, Paris, Presses de Sciences Po, 2011 ;
} 
montrer qu'il existe bien des formes de gouvernance dans les grandes métropoles, mais qu'il s'agit du résultat de bricolages ad hoc, et d'improvisation pragmatique, combinant différentes formes d'agences, des réseaux et de formes souples de gouvernance.

Au cours du $20^{\mathrm{e}}$ siècle, dans le cadre des États, et sous leur contrôle, les dirigeants des villes ont accru leurs capacités gouvernementales formelles. Mais progressivement, ils ne se sont plus contentés de seulement mettre en œuvre les règles ou les politiques définies par les gouvernements. Ils créent les leurs. En outre, les villes ont généré de nombreux processus politiques non gouvernementaux qui organisent la vie dans la ville. Le terme " gouvernance » est utilisé pour désigner ces autres façons d'ordonner les villes ${ }^{59}$. Il s'agit notamment de coalitions et de réseaux informels, ainsi que de normes informelles largement suivies, créant des formes d'autorité efficaces qui dépassent les pouvoirs du gouvernement lui-même. Ces normes, réseaux, coalitions et pratiques peuvent également donner forme à des coalitions qui façonnent les politiques publiques et le gouvernement formels. Elles orientent le débat public sur les objectifs et l'avenir.

Contrairement aux travaux classiques sur le gouvernement urbain et les -urban politics-(cf. infra), avec Tommaso Vitale ${ }^{60}$, nous essayons de développer une sociologie politique critique de la gouvernance arguant que les villes sont plus ou moins gouvernées, avec une intensité différente dans l'espace et dans le temps. La sociologie politique de la gouvernance tente de combiner l'analyse des activités et des institutions du gouvernement, de l'agence des acteurs, des limites du gouvernement et de la manière dont les formes d'ordre social et politique s'articulent dans les modes de gouvernance, y compris les acteurs non gouvernementaux et la capacité à créer des incitations, des normes, des règles, voire des conventions ou des certifications pour mettre en œuvre des politiques. Elles sont souvent un lieu de privation et de violence et l'absence de gouvernance classique dans les périphéries de ces villes est dramatique pour les populations pauvres car la violence devient l'alternative à des formes « parallèles » d'ordre, de contrôle, de distribution des ressources, de légitimité et d'identité. Dans les grandes villes pauvres, plus encore que dans d'autres contextes, les échecs de politiques publiques sont la norme, les gouvernements n'obtiennent souvent pas grand-

\footnotetext{
Jean-Marc Offner et Frédéric Gilli, Paris, métropole hors les murs. Aménager et gouverner un Grand Paris, Paris, Presses de Sciences Po, 2009, p. 200.

${ }^{59}$ Olivier Borraz et Patrick Le Galès, « Urban Governance in Europe: The Government of What? », Pôle Sud, vol. $1, \mathrm{n}^{\mathrm{o}} 32,2010$, p. 137-151.

${ }^{60}$ Patrick Le Galès, Tommaso Vitale, « Diseguaglianze e discontinuità nel governo delle grandi metropoli. Un'agenda di ricerca ». Territorio. p.7-17, 2015
} 
chose, les inégalités restent massives, les arrangements informels sont déterminants ${ }^{61}$. JulieAnne Boudreau ${ }^{62}$ souligne avec raison que l'informel et l'illégal sont des parties constituantes du politique dans les villes.

La gouvernance métropolitaine n'est ni un processus linéaire ni un processus rationnel ; elle est incomplète et sujette à des discontinuités, centrée sur la résolution de conflits entre groupes inégaux en compétition ${ }^{63}$. La vie urbaine est caractérisée par la prolifération des formes de gouvernance et des pratiques d'autonomie, qui génèrent un ordre instable et non susceptible d'un contrôle total par l'État, le marché, la religion ou autre. Une politique de l'urbain doit faire face à cette complexité. Le désordre des uns peut être la gouvernance cohérente des autres. La ville ségréguée peut être organisée et bien gouvernée d'un point de vue, mais fondamentalement violente en refusant des opportunités à de larges sections de la population urbaine. En complément des travaux notamment des anthropologues, le côté illégal ou informel de la gouvernance urbaine nous paraît aussi important que le gouvernement; à savoir le rôle des gangs, des mafias, des églises (églises évangélistes dans les bidonvilles par exemple), des groupes de vigilantisme ${ }^{64}$, de la corruption, des activités informelles peu gouvernées de manière légale.

L'échelle, la portée et la variété des processus d'urbanisation du $21^{\mathrm{e}}$ siècle augmentent la variété des formes de gouvernance non traditionnelles ${ }^{65}$. La plupart des zones métropolitaines présentent une combinaison de désordres dus par exemple aux gangs, au trafic de drogue, ou bien aux infrastructures dysfonctionnelles et aux services qui ne fonctionnent pas. Mais il existe également d'autres formes d'ordre dans les villes du monde entier, comme la collecte des déchets (et des marchés de recyclage dynamiques) au Caire, l'organisation de systèmes de bus informels à Nairobi ou à Lusaka, la médiation entre la police et les habitants pour réduire la criminalité à Chicago, la fourniture de services de santé de proximité à Los Angeles ou à Naples, les soutiens de la mafia à des populations en difficulté. Parmi les acteurs qui produisent des régulations et des services, dans des endroits comme São Paulo, Le Cap ou Mumbai, mais aussi Montréal, New York ou Paris figurent le crime organisé, les églises, certains syndicats, des chefs d'entreprise et des promoteurs privés, ainsi que les

\footnotetext{
${ }^{61}$ Kees Koonings et Ditk Kruijt (dir.), Megacities: The Politics of Urban Exclusion and Violence in The Global South, Londres/New York, Zed Books, 2009.

${ }^{62}$ Julie-Anne Boudreau, Global Urban Politics, op. cit.

${ }^{63}$ Patrick Le Galès, « Urban governance in Europe: What is governed?". The new blackwell companion to the city, 747-758. 2011

${ }^{64}$ Gilles Favarel-Garrigues et Laurent Gayer, « Violer la loi pour maintenir l'ordre. Le vigilantisme en débat », Politix, vol. 3, n 115, 2016, p. 7-33 ; Jennifer M. Hazen et Dennis Rodgers (dir.), Global Gangs: Street Violence Across The World, Minneapolis/Londres, University of Minnesota Press, 2014.

65_Warren Magnusson, Politics of Urbanism..., op. cit.
} 
mafias locales ${ }^{66}$.

Enfin, dans les métropoles, la politique est sans doute plus qu'au niveau national structurée par les politiques publiques, pour reprendre l'intuition initiale de Ted Lowi ${ }^{67}$. Les villes doivent faire face à des problèmes d'action collective qui donnent lieu à d'importantes politiques publiques notamment en termes de sécurité, de lutte contre la pauvreté, d'accueil ou de rejet des migrants, de mobilité, d'environnement, de développement économique, de culture, de logement, d'urbanisme ${ }^{68}$. La mise en œuvre des politiques publiques urbaines produit des contestations, de nouvelles définitions des buts à atteindre, de l'enrôlement et de l'organisation d'acteurs qui, en retour, organisent la politique. Plus les politiques publiques sont importantes par les budgets, les normes, les instruments, les résultats et plus la politique est définie à partir des politiques publiques et de leurs résultats. Ces questions se posent dans la majorité des métropoles et des villes dans le monde. Les processus de globalisation font que les entreprises, les ONG, les organisations internationales (UN-Habitat ou la Banque mondiale), les associations d'élus, les consultants, les universitaires font partie d'un monde globalisé des politiques urbaines ou circulent des idées, des recettes, des capitaux, des solutions, des instruments, des statistiques, des technologies, des normes, des professions, bien au-delà du contrôle des États. En d'autres termes, les planners post-modernes ont beau dénoncer de manière rituelle l'ingouvernabilité des métropoles, des lignes de bus et de métros sont construits, des quartiers et des logements sortent de terres, des polices maintiennent un certain ordre (plus ou moins corrompu ou violent), des politiques de redistribution sont mises en œuvre, des politiques de peuplement et de ségrégation excluent ou intègrent des groupes et des migrants, des réseaux d'eau sont connectés, des groupes plus ou moins légaux gèrent des ordures et tout ceci se mesure à l'aune des budgets et par des groupes qui protestent et qui contestent ${ }^{69}$ et par des projets urbains multiples qui sont de puissants instruments de délibération sur les objectifs, sur l'organisation de l'action collective, la résolution des

\footnotetext{
${ }^{6}{ }^{6}$ Jo Beall, Tom Goodfellow, Dennis Rodgers, « Cities and Conflict in Fragile States in the Developing World », Urban Studies, vol. 50, n 15, 2013, p. 3065-3083 ; Cesare Mattina, Clientélismes urbains. Gouvernement et hégémonie politique à Marseille, Paris, Presses de Sciences Po, 2016.

${ }^{67}$ Theodore J. Lowi, « Four Systems of Policy, Politics, and Choice », Public administration review, vol. 32, $\mathrm{n}^{\circ}$ 4, 1972, p. 298-310 ; Hubert Heinelt, « Do Policies Determine Politics? », in Frank Fischer, Gerald J. Miller, Mara S. Sidney (dir.), Handbook of Public Policy Analysis, Londres/New York, Routledge, 2017, p. 135-146. ${ }^{68}$ Altison E. Post, « Cities and Politics in the Developing World», art. cité-

${ }^{69}$ Bekker Laurent Fourchard, op.cit (S'agit-il de: Laurent Fourchard, Trier, exclure et policer..., op. cit.?) ; Côme Salvaire, « From Urban Congestion to Political Confinement: Collecting Waste, Channelling Politics in Lagos », Territory, Politics, Governance, vol. 8, n 1, 2019, p. 1-17 ; Joël Ruet et Stéphanie Tawa Lama-Rewal (dir.), Governing India's Metropolises: Case Studies of Four Cities, , New Delhi/Abingdon, Routledge, 2012. Paul Kantor, Christian Lefèvre, Asato Saito, Hank V. Savitch, Andy Thornley, Struggling Giants: City-Region Governance in London, New York, Paris, and Tokyo, Minneapolis/Londres, University of Minnesota Press, 2012.
} 
conflits, l'enrôlement d'acteurs et l'exercice d'une contrainte ${ }^{70}$.

Ce point nuance l'analyse de Magnusson. Malgré tout l'intérêt de sa théorisation, son analyse a tendance à définir l'État de manière étroite et un peu caricaturale. Or les travaux sur l'État ont montré la déconnexion entre territoires et État, entre État et nation, et les processus de dénationalisation de l'autorité politique ou le sort contrasté de différents éléments qui rentrent dans le concept de souveraineté. Comme souvent, Magnusson met surtout l'accent sur la délibération, la démocratie, et assez peu sur les résultats de l'action des gouvernements quels qu'ils soient. Par conséquent, à part en termes de domaine d'action, une partie des politiques menées par les élites urbaines ne sont pas très différentes des élites étatiques. Le politique des villes et des métropoles est en partie inscrit au sein des États (y compris en opposition). Cependant, dans de nombreux cas, les villes ou les métropoles au sens d'acteurs collectifs très incomplets et traversés par des conflits ont gagné en autonomie, en capacité stratégique, en capacité de mise en œuvre des politiques publiques et de résolution de conflits, en termes de gouvernance donc. La politique des villes semble, parfois, s'autonomiser, se différencier de celle des États par exemple sur les questions de participation, de démocratie locale, d'accueil de migrants ou de lutte contre le changement climatique.

Les élites de certaines villes ou métropoles poussent à l'extrême la surveillance et la relégation des pauvres, la privatisation des biens communs et la spéculation urbaine. D'autres inventent des formes progressives de démocratie locale radicale (à Barcelone ou Madrid), de gestion des ressources naturelles ou d'intégration des migrants. La différenciation des villes se voit aussi en termes sociaux : des services, des modes de vie, des types de populations se concentrent dans les villes et les métropoles ce qui conduit à des comportements politiques parfois différenciés (des valeurs plus libérales ou cosmopolites) ${ }^{71}$. Ceci fait écho aux travaux de Iris Marion Young ${ }^{72}$ sur une théorie spatialisée de la justice qui caractérise la politique démocratique de la ville comme la prise en compte de la parole des différents groupes juxtaposés dans une ville, sans faire communauté, sans domination de l'un d'entre eux. Son idéal de ville démocratique repose sur la prise en compte pragmatique de ces relations entre groupes sociaux différenciés, mais qui ne conduit ni à la ségrégation spatiale ou à l'exclusion ni au renfermement sur des communautés.

Cette sociologie politique de la gouvernance urbaine interroge également la démocratie

\footnotetext{
${ }^{70}$ Gilles Pinson, Gouverner par projet. Urbanisme et gouvernance des villes européennes, Paris, Presses de Sciences Po, 2009.

${ }^{71}$ Mike Raco, « Critical Urban Cosmopolitanism and The Governance of Urban Diversity in European Cities », European Urban and Regional Studies, vol. 25, $\mathrm{n}^{\circ}$ 1, 2018, p. 8-23 ; Michael Keith, After the Cosmopolitan?: Multicultural Cities and the Future of Racism, Londres/New York, Routledge, 2005.)

${ }^{72}$ Iris Marion Young, Justice and the Politics of Difference, op. cit.
} 
urbaine. De nombreux travaux utilisent le laboratoire urbain pour identifier des formes de démocraties, ou expérimenter des dispositifs à partir de communautés, de quartiers, de budget, de plateforme numérique ${ }^{73}$ et développer une réflexion critique sur les limites de la démocratie urbaine qui va à l'encontre des travaux classiques sur le gouvernement local.

Constatant la tension entre multiculturalisme, diversité et solidarité au niveau national, Will Kymlicka se demande si les villes ne pourraient pas inventer des formes différentes de solidarité ${ }^{74}$ intégrant les migrants et renforcées par des politiques publiques urbaines et le droit de vote des étrangers, ce qui réactualiserait une forme de républicanisme radical urbain ${ }^{75}$. Contrairement à ce qu'on voit à l'échelle nationale, les habitants des métropoles sont plus enclins à soutenir à la fois le développement de politiques sociales redistributives et l'accueil de migrants et de réfugiés. Rainer Bauböck envisage des formes de citoyenneté urbaine complémentaires de la citoyenneté nationale pour donner des droits aux personnes de passages, aux mobiles, aux migrants ${ }^{76}$.

\section{Des villes justes et démocratiques ?}

Le cycle contemporain mondial d'urbanisation massive donne lieu à des débats théoriques majeurs, disputés, à une inflation de concepts pour rendre compte du nouveau monde urbain. Ce débat interdisciplinaire est dominé par les géographes et les urbanistes qui s'inscrivent dans l'héritage d'Henri Lefebvre" ${ }^{77}$, et notamment le monde bigarré des critical urban scholars $^{78}$ néo-marxistes ou post-marxistes qui rivalisent d'imagination et d'emprunts

\footnotetext{
${ }^{73}$ Arjun Appadurai, « Deep Democracy: Urban Governmentality and the Horizon of Politics », Environment and Urbanization, vol. 13, n 2, 2001, p. 23-43; Archon Fung, Empowered Participation: Reinventing Urban Democracy, Princeton, Princeton University Press, 2009. Gianpaolo Baiocchi, Patrick Heller, Marcelo K. Silva, Bootstrapping Democracy: Transforming Local Governance and Civil Society in Brazil, Stanford, Stanford University Press, 2011 ; Oscar W. Gabriel, Vincent Hoffmann-Martinot, Hank V. Savitch (dir.), Urban Democracy, Berlin, Springer Science \& Business Media, 2012.

${ }^{74}$ Keith Banting et Will Kymlicka (dir.), The Strains of Commitment: The Political Sources of Solidarity in Diverse Societies, Oxford/New York, Oxford University Press, 2017.

${ }^{75}$ Jonathan Matthew Hoye, « Sanctuary Cities and Republican Liberty », Politics \& Society, vol. 48, n 1, 2020, p. 67-97.

${ }^{76}$ Rainer Bauböck, « Cities vs States... », art. cité ; Lynn A. Staeheli, « Cities and citizenship », Urban Geography, vol. 24, n 2, 2003, p. 97-102 ; Andrew Linklater, The Transformation of Political Community, Cambridge, Polity, 1998 ; Rinus Penninx, Karen Kraal, Marco Martiniello, Steven Vertovec, Citizenship in European Cities: Immigrants, Local Politics and Integration Policies, Londres/New York, Routledge, 2017. ${ }^{77}$ Plusieurs manuels consacrés à Urban Politics sont en fait écrits par des géographes critiques nord-américains ou britanniques par exemple Mark Davidson et Deborah Martin (dir.), Urban Politics: Critical Approaches, Los Angeles/Londres, Sage, 2013.

${ }^{78}$ Michael Storper, « The Poverty of Radical Theory Today: From the False Promises of Marxism to the Mirage of the Cultural Turn », International Journal of Urban and Regional Research, vol. 25, nº 1, 2001, p. 155-179; Jonathan Metzger, Philip Allmendinger, Stijn Oosterlynck (dir.), Planning Against the Political, Londres, Routledge, 2015.
} 
philosophiques $^{79}$. L'une des caractéristiques de ces débats théoriques de critical urban studies $^{80}$ est parfois la marginalisation de la question politique.

\section{Droit à la ville}

Pour la géographie urbaine critique, la dynamique massive dite -d' « urbanisation planétaire $^{81}$ » conduit à l'obsolescence de conceptions classiques de l'État, des institutions, des partis, des élections, des politiques publiques, de la démocratie représentative ou de la citoyenneté. Cette veine donne lieu à une production intellectuellement stimulante, pour penser the urban after the age of the city ${ }^{82}$. Ces géographes critiques dénoncent avec vigueur le methodological cityism, c'est-à-dire le fait qu'une partie de la recherche urbaine s'appuie encore sur des conceptions de villes ou de métropoles. De nombreux auteurs soulignent l'extension incessante des banlieues, le développement de «non-lieux ${ }^{83} »$, d'espaces urbains anonymes similaires (bretelles d'autoroute, centres commerciaux, lotissements résidentiels, zones de loisirs marchandisées, parkings, gares et aéroports, immeubles de bureaux et parcs de loisirs) et de mégalopoles («post-villes ») dans différentes parties du monde. Dans cette ligne d'analyse, la dissolution de la ville a lieu dans un grand monde urbain fragmenté, chaotique et instable. C'est l'époque des « citoyens sans villes ${ }^{84} »$ où de nouvelles formes et de nouvelles expériences sont inventées. Certains architectes et urbanistes, par exemple Rem Koolhaas, se précipitent avec passion vers une sorte de monde urbain, apparemment libéré des contraintes classiques (de l'État, des règles, de la lenteur, du substrat social, de la fixité, des conflits sociaux). Ils sont éblouis par la vitesse, la fluidité et les échelles d'urbanisation des mégalopoles asiatiques ou africaines, par une globalisation de la pensée urbaine innovante à la

\footnotetext{
${ }^{79}$ Dans son article, « What Do Cities Have to Do with Democracy? » (International Journal of Urban and Regional Research, vol. 38, $\mathrm{n}^{\circ}$ 5, 2014), Clive Barnett ironise sur « les travaux théoriques liés à l'effondrement du marxisme orthodoxe - je pense notamment aux enquêtes et spéculations délicieusement variées de penseurs français comme Bachelard, Badiou, Bataille, Barthes, Baudrillard, Bourdieu, Clastres, Debord, Deleuze, Derrida, Foucault, Lacan, Latour, Lefort, Levinas, Levi-Strauss, Lyotard et Rancière, mais la liste pourrait être allongée et diversifiée - et imposer une compréhension de la politique urbaine qui - bien que perspicace à bien des égards est en contradiction avec l'expérience de la plupart des gens » : p. 1625-1643 (notre traduction).

${ }^{80}$ Peter Marcuse, David Imbroscio, Simon Parker et al., « Critical Urban Theory Versus Critical Urban Studies: A Review Debate », International Journal of Urban and Regional Research, vol. 38, n 5, 2014, p. 1904-1917. Warren Magnusson, « The Symbiosis of the Urban and the Political », International Journal of Urban and Regional Research, vol. 38, n 5, 2014, p. 1561-1575.

${ }^{81}$ Neil Brenner et Christian Schmid, «Planetary Urbanization », in Xuefei Ren et Roger Keil (dir.), The Globalizing Cities Reader, Routledge, 2017, p. 479-482.

${ }^{82}$ Engin Isin, « City.state: Critique of Scalar Thought », Citizenship Studies, vol. 11, n 2, 2007, p. 211-228 ; Lauren Rickards, Brendan Gleeson, Mark Boyle, Cian O’Callaghan, « Urban Studies after the Age of the City », Urban Studies, vol. 53, n 8, 2016, p. 1523-1541 ; Hillary Angelo et David Wachsmuth, « Urbanizing Urban Political Ecology: A Critique of Methodological Cityism », International Journal of Urban and Regional Research, vol. 39, $\mathrm{n}^{\circ} 1,2015$, p. 16-27.

${ }^{83}$ Marc Augé, Non-lieux. Introduction à une anthropologie de la surmodernité, Paris, Seuil, 2015.

${ }^{84}$ Michel Agier, L'invention de la ville : banlieues, townships, invasions et favelas, Paris, La documentation française, 1999.
} 
pointe du cyber-monde, et par l'invention de formes réalisables grâce aux technologies.

La nécessité de penser cette urbanisation au-delà des villes a conduit à des travaux particulièrement novateurs et importants. Parfois cependant, cette approche radicale tend à faire table rase du passé, à ignorer ou à éliminer la recherche en science sociale au profit de perspectives moins ancrées dans la recherche empirique ${ }^{85}$. Par exemple, le travail original de théorisation des géographes urbains post-modernistes de UCLA faisait de Los Angeles l'avenir du monde urbain généralisé et fractalisé, un monde urbain sans politique, ce qui s'est révélé une double erreur : les transformations de Los Angeles l'ont beaucoup rapproché d'autres métropoles mondiales et d'autre part la politique classique y joue un rôle central depuis que des démocrates se mobilisent en faveur de politiques redistributives et $\mathrm{du}$ renforcement des services publics. Enfin, certains spécialistes qui se présentent comme urban scientists, souvent des physiciens, affirment que toutes les villes et tous les processus d'urbanisation découlent de lois d'échelle universelles qui s'appliquent à tous les contextes et à toutes les époques. Cette approche mobilise les métadonnées avec le rêve d'algorithmes modélisant et mesurant toutes les interactions et tous les événements ce qui pourrait permettre une gestion urbaine optimale, automatique, efficace, reposant sur des données, non conflictuelles, c'est-à-dire le rêve scientiste ou technocratique logiquement absurde de la poleis sans politique ${ }^{86}$.

Au contraire, un courant d'urbanistes et de géographes critiques a entrepris de repenser le politique urbain à partir de perspectives normatives de justice (la ville juste ${ }^{87}$ ), mais aussi d'un rejet et d'une ignorance : le rejet de la politique institutionnelle et l'ignorance d'une grande partie de la science politique. Dans un ouvrage fameux de 1968, Henri Lefebvre avait théorisé « le droit à la ville ${ }^{88} »$ devenu concept normatif, slogan révolutionnaire ou programme de politique urbaine. Il dénonçait dans ses ouvrages l'emprise des bureaucraties (y compris communistes), du capitalisme et leur logique d'exclusion. Il se faisait le chantre d'une vision radicalement politique d'une société urbaine démocratique autogérée par la population. Une

\footnotetext{
${ }^{85}$ Edward W. Soja, Thirdspace: Expanding the Geographical Imagination, Oxford, Wiley-Blackwell, 1996 ; Michael Dear (dir.), From Chicago to LA: Making Sense of Urban Theory, Thousand Oaks/Londres/new Delhi, Sage, 2002.

${ }^{86}$ Michael Batty, The New Science of Cities, Cambridge, MIT Press, 2013.

${ }^{87}$ Susan. S. Fainstein, « The Just City », International Journal of Urban Sciences, vol. 18, nº 1, 2014, p. 1-18. David Harvey, Social Justice and the City, Athens/Londres, University of Georgia Press, 2010.

${ }^{88}$ Henri Lefebvre, Le droit à la ville, Paris, Economica-Antrhopos, 1968. Voir aussi David Harvey, « The Right to the City », International Journal of Urban and Regional Research, vol. 27, n 4, 2003, p. 939-941 ; Donovan Mitchell, The Right to the City: Social Justice and the Fight for Public Space, New York/Londres, Guilford Press, 2003 ; Mark Purcell, « Citizenship and the Right to the Global City : Reimagining the Capitalist World Order », International Journal of Urban and Regional Research, vol. 27, n 3, 2003, p. 564-590. Bernard Bret et al. (dir.), Justice et injustices spatiales, Paris, Presses universitaires de Paris Ouest, 2010 ; Kurt Iveson, « Social or spatial justice? Marcuse and Soja on the right to the city », City, vol. 15, n 2, p. 250-259.
} 
société juste, démocratique, organisée autour des besoins des habitants, pouvait être envisagée à l'échelle de la ville. Les habitants devaient pouvoir « produire» la ville, perspective devenue centrale dans les travaux de géographes critiques états-uniens et britanniques. Cette question du droit à la ville est de plus en plus posée dans les villes africaines ou asiatiques en termes de citoyenneté : une « citoyenneté urbaine, distincte et indépendante de la citoyenneté nationale, qui donnerait accès à des droits élémentaires et énumérables à être en ville. Cela se traduirait a minima en termes d'accès à un logement, à un emploi et à la mobilité. La citoyenneté urbaine rime aussi avec droit à la participation aux arènes du débat politique local ${ }^{89}$. $\gg$ Cette question de citoyenneté urbaine plus ou moins combinée ou complémentaire de la conception nationale de la citoyenneté a été mise en avant par les travaux qui s'intéressent aux droits des migrants légaux ou illégaux et des réfugiés qui sont massivement concentrés dans les villes ${ }^{90}$.

Ces travaux ont été poursuivis par des réflexions sur le principe de justice spatiale pensé en relation avec la lutte des classes dans la ville, contre l'urbanisation capitaliste, la gentrification, les ghettos de riches (gated communities) ${ }^{91}$, la fermeture et le contrôle des espaces publics, la surveillance policière soit pour mobiliser des coalitions de gauche et changer la ville soit pour préparer la révolution anti-capitaliste dans la version de David Harvey ${ }^{92}$. Dans ces travaux, l'État est toujours l'ennemi au service du capital et seuls les mouvements sociaux radicaux peuvent permettre une transformation sociale radicale. Le politique dans la ville c'est la mobilisation contre le capitalisme, et la révolution à venir.

\section{La ville post-politique}

Dans un registre comparable, un courant de recherche très dynamique a émergé des travaux d'un groupe de philosophes et de géographes mobilisés par Erik Swyngedouw qui s'inscrit dans le cadre de la « post-politique ». Ignorant encore une fois les recherches en science politique, ces chercheurs s'intéressent aux limites des régulations politiques, aux menaces qui pèsent sur la démocratie, à l'impact de l'inégalité de la gouvernance démocratique et à la transformation des villes. Erik Swyngedouw et Japhy Wilson s’inspirent

\footnotetext{
${ }^{89}$ Marianne Morange et Amandine Spire, « Mise en ordre, mise aux normes et droit à la ville : perspectives croisées depuis les villes du Sud », Métropoles, n 21, 2017.

${ }^{90}$ Avner de Shalit, Cities and Immigration. Political and Moral Dilemmas in the New Era of Migration, Oxford, Oxford University Press, 2018. Karen Kraal, Steven Vertovec, (dir.) Citizenship in European Cities, Abingdon, Routledge, 2017

${ }^{91}$ Teresa P. R. Caldeira, City of Walls: Crime, Segregation, and Citizenship in São Paulo, University of California Press, 2000.

${ }^{92}$ Neil Brenner, Peter Marcuse et Margit Mayer (dir.), Cities for People, Not for Profit: Critical Urban Theory and the Right to the City, Abingdon, Routledge, 2012.
} 
de Jacques Rancière et décrivent une tendance dans laquelle « le politique - compris comme un espace de contestation et d'engagement agonistique - est de plus en plus colonisé par la politique - compris comme des mécanismes technocratiques et des procédures consensuelles qui fonctionnent dans un cadre incontesté de démocratie représentative, d'économie de marché libre et de libéralisme cosmopolite. Dans la post-politique, les contradictions politiques sont réduites à des problèmes politiques qui doivent être gérés par des experts et légitimés par des processus participatifs dans lesquels la portée des résultats possibles est étroitement définie à l'avance. Le " peuple " - en tant que collectif politique potentiellement perturbateur, est remplacé par la population - objet agrégé des sondages d'opinion, de la surveillance et de l'optimisation biopolitique. Les citoyens deviennent des consommateurs, et les élections sont conçues comme un “ choix" parmi d'autres, dans lequel les individus choisissent en privé leurs gestionnaires préférés des conditions de nécessité économique ${ }^{93}$. »

Ce groupe de géographes radicaux part d'une critique convaincante des limites de la démocratie, des oligarchies urbaines et des élections par rapport à la question environnementale ${ }^{94}$. Ils visent à identifier la « disparition du politique dans les arrangements post-politiques », afin d'ouvrir la voie à de nouvelles possibilités et au retour du politique. S'appuyant sur les travaux de philosophes dits de la « démocratie radicale » (Chantal Mouffe, Jacques Rancière, Slavoj Žižek et Alain Badiou), ils espèrent définir ce qui apparaît comme « proprement politique », avec un rejet des démocraties libérales, des gouvernements, et une ignorance des politiques publiques, de l'action collective, des institutions, des dispositifs, du droit qui, parfois, sont aussi des armes qui modifient les rapports de pouvoir.

Pour ces géographes critiques, la politique contemporaine est uniquement définie en termes de « nouvelle gestion publique », de « bonne gouvernance » ou de transfert des « meilleures pratiques », tout ceci caractérisant le domaine de la science politique. Comme si les politistes n'avaient jamais traité des conflits, de la violence, de la concurrence politique, de la domination depuis Machiavel ou Hobbes. La littérature sur la «post-politique » soutient que la politique normale est une question de dépolitisation, de règne des experts et d'exclusion des citoyens, laissant de côté la tension permanente entre la tentative de créer un certain ordre politique, de créer un consensus, de créer des formes de bien commun et d'intérêts généraux, de maintenir la domination de certains groupes contre les mobilisations sociales, les contestations, les groupes d'intérêts en concurrence permanente, la violence et les

\footnotetext{
${ }^{93}$ Erik Swyngedouw et Japhy Wilson, The Post-Political and Its Discontents. Spaces of Depoliticisation, Spectres of Radical Politics, Édimbourg, Edinburg University Press, 2014, p. 16-17?

${ }^{94}$ Erik Swyngedouw, « The Zero-Ground Of Politics: Musings On The Post-Political City », New Geographies, vol. $1, \mathrm{n}^{\circ} 1,2009$, p. 52-61.
} 
guerres, et la mise en œuvre de politiques toujours transformées et contestées. La notion d'un « arrangement post-politique » est une perspective intéressante pour étudier précisément les processus de dépolitisation (qui ont toujours été en jeu), et qui peuvent être plus saillants dans certains domaines et dans certaines villes. Cependant, ce que l'on appelle « post-politique » ou « dépolitisation » est la plupart du temps une « politisation » différente, favorisant différents groupes et promue par certaines coalitions.

Le débat sur la post-politique est une discussion philosophique visant à « recentrer » le politique indépendamment de la question de savoir ce qui constitue la politique qui rappelle un peu le débat entre Jacques Rancière et Pierre Bourdieu récemment réexaminé par Côme Salvaire ${ }^{95}$. En ce sens, il s'agit d'une entreprise intellectuelle au sein d'une ontologie particulière. Elle conduit à une vision prophétique de la politique définie autour de questions environnementales, qui doit s'exprimer dans des mouvements révolutionnaires.

En d'autres termes, l'arrangement post-politique prétendument consensuel est une notion écran. Le recours systématique aux formulations en termes de « fin du dissensus », ou « fin de la politique » parait pour le moins contredit par la dynamique actuelle des mobilisations politiques dans de nombreuses villes, par les conflits autour de la fiscalité et de la corruption, les conflits religieux, les attaques terroristes, les maires de gauche fournissant des services dans les villes d'Amérique latine, ou la montée de la droite radicale dans les villes américaines et européennes. Ce ne sont pas des signes de dépolitisation. La démocratisation est toujours incomplète et contestée, la dépolitisation renvoie à une série de processus qui doivent être conceptualisés et examinés de manière empirique. Le projet « postpolitique » s'appuie sur les travaux de Jacques Rancière pour recentrer la politique sur des valeurs d'égalité, de liberté et de démocratie tout en accentuant l'aspect contingent et fluide de l'ordre social et politique. On retrouve la contradiction classique entre un hyper constructivisme d'une part et la dénonciation de la domination de groupes et d'institutions qui bloquent l'émergence de la vraie politique. Il s'agit d'un projet intellectuel normatif dans l'attente d'une mobilisation pour transformer l'ordre politique existant. Clive Barnett synthétise bien le problème : «"post-politique” et "ville post-démocratique” ne peuvent qu'invoquer des idéaux ontologisés ahistoriques sur ce que devrait être la politique démocratique afin de déterminer qu'en réalité, elle ne s'accorde pas à ces idéaux ${ }^{96} \gg$.

\footnotetext{
${ }^{95}$ Côme Salvaire, « Recovering Sociology from the Trash Heap: Of Waste Politics and the Spatialization of Local Representation in Lagos », International Journal of Urban and Regional Research, vol. 43, n 2, 2019, p. 354-376.

${ }^{96}$ «"post-political” and "post-democratic city” can do no more than invoke ahistorical ontologized ideals about what democratic politics should be like in order to determine that, in reality, it does not accord to these ideal" $\gg$ :
} 


\section{L'urbain comme système socio-technique et la politique pragmatique}

Très influencé par Bruno Latour et Michel Foucault, un courant de recherche urbaine a combiné sociologie des sciences et des technologies, cultural studies, pragmatisme, philosophie post-structuraliste, études post-coloniales et urbanisme (et parfois l'anthropologie) pour penser le politique urbain indépendamment des approches plus traditionnelles en termes d'institutions, de ressources, de mobilisation, de relation de pouvoir, de communauté politique, de coercition ou d'autonomisation relative d'un domaine particulier d'activité sociale ${ }^{97}$.

La ville, l'urbain sont d'abord pensés à partir des infrastructures, des technologies, des données. Les infrastructures, les réseaux d'eau, d'énergie, de transport, de déchet, de données sont une partie constituante des villes depuis leur origine, ce qui rappelle les travaux anciens du Centre d'étude de recherche et de formation institutionnelles (CERFI) et de Michel Foucault sur les équipements du pouvoir dans les villes ${ }^{98}$. Avec la Révolution industrielle, ces dimensions sont devenues essentielles pour les métropoles industrielles et les capitales d'empire de la fin du 19e siècle comme Londres, Paris, Berlin, Vienne puis New York. Dans cette perspective, le politique, les politiques publiques, la gouvernance sont largement déterminées par les infrastructures. Le politique urbain, envisagé d'abord dans un cadre d'abord municipal puis national, est conçu en termes d'investissements publics, de partenariat public-privé, d'espace public, de corruption, nécessaires pour construire et maintenir les infrastructures et les réseaux indispensables à l'existence de ces métropoles et le bon fonctionnement de leur métabolisme. Dans cette première conceptualisation, les infrastructures et les réseaux sont cependant le résultat de conflits, de relation de pouvoir, un système de représentation qui progressivement structure le politique urbain.

La révolution industrielle en cours (financière et technologique) fait des infrastructures urbaines l'un des points centraux du capitalisme contemporain. Inspirés par la théorie de l'acteur réseau de Michel Callon et Bruno Latour, tout un ensemble d'auteurs a tenté de conceptualiser un système socio-technique urbain, une cyborg city caractérisée par un assemblage complexe, pluriel, hybride de réseaux, de réseaux technologiques, d'organisations, de machines, d'acteurs humains et non humains, d'interactions entre ces

\footnotetext{
Clive Barnett, « What do cities have to do with democracy? », International Journal of Urban and Regional Research, vol. 38, n 5, 2014, p. 1625-1643, p.1629 (notre traduction).

${ }^{97}$ Bruno Latour et Émilie Hermant, Paris ville invisible, Les Empêcheurs de Penser en Rond/La Découverte, Paris, 1998.

${ }^{98}$ Voir le numéro spércial de Recherches, « Généalogie du capital.1 les équipements du pouvoir, villes territoires et équipements collectifs », 1973, n.13. Je remercie Pierre Lascoumes pour cette référence et pour sa mémoire.
} 
éléments et doté d'une certaine capacité d'action ${ }^{99}$. Reprenant les développements de Gilles Deleuze et Félix Guattari sur le rhizome, l'urbain est conceptualisé comme fluide, en mouvement, indéterminé, toujours unique ${ }^{100}$. De manière décisive, ces auteurs mettent en avant l'hybridité de la ville et le politique au-delà du citoyen ou de l'humain, c'est à dire le discours, le politique post-humain articulant (un mot clé avec hybridité) humain, technologies, réseaux. « La ville cyborg est largement perçue comme une ville post-métabolique dans laquelle l'échange d'informations a supplanté le rôle de l'échange matériel pour devenir la dynamique dominante derrière le façonnage de l'espace urbain ${ }^{101}$. » D'un côté, l'ordre dans la ville est pensé à partir des réseaux et des infrastructures, de l'autre, la multiplicité des interactions entre ces différentes composantes et les expériences possibles créent des formes structurelles d'indétermination de la ville, des formes spatiales et un monde d'imagination et de possibles. Ces systèmes socio-techniques urbains sont dotés d'une capacité d'action, de décision qui font écho à toute une littérature de science-fiction, par exemple les romans de William Gibson ${ }^{102}$. Ces assemblages laissent prise à des trous noirs, une myriade d'activités informelles ou illégales, des interstices, des territoires hors contrôle ainsi qu'à des capacités de contrôles, des oligarchies, des capacités de surveillance contrôlées par des acteurs privés. Le politique urbain est alors défini en termes d'espace public à construire et à défendre autour et avec les infrastructures et les réseaux ${ }^{103}$, comme un monde de possible, virtuel ou réel, imprévisible. Suivant l'intention de Dewey, cet assemblage urbain crée ses publics.

Dans cette même veine, Ash Amin et Nigel Thrift ${ }^{104}$ ont proposé, dans leurs différents ouvrages, des conceptualisations de la ville et du politique. Comme tous les géographes critiques, leur point de départ est radical et furieusement complexe ! « la ville est partout et dans tout ... Elles [les villes] soutiennent des pratiques sociales d'une diversité inimaginable ${ }^{105}$. » Ils proposent une analyse du politique qui n'écarte pas les questions de

\footnotetext{
${ }^{99}$ Antoine Picon, La ville, territoire des cyborgs, Besançon/Paris, Les Éditions de L'Imprimeur, 1998. Christine M. Boyer, Cybercities: Visual Perception In The Age Of Electronic Communication, New York, Princeton Architectural Press, 1996. Matthew Gandy, « Cyborg Urbanization: Complexity and Monstrosity in the Contemporary City », International Journal of Urban and Regional Research, vol. 29, n 1, 2005, p. 26-49. ${ }^{100}$ Ash Amin et Nigel Thrift, Cities: Reimagining the Urban, Bristol, Polity Press, 2002.

${ }^{101}$ « The cyborg city is widely perceived as a post-metabolic city in which the exchange of information has supplanted the role of material exchange to become the dominant dynamic behind the shaping of urban space »: «Cyborg Urbanization... », art. cité, p. 35 (notre traduction).

${ }^{102}$ Popularisés notamment par le film Matrix réalisé par les Wachowski en 1999.

${ }^{103}$ Voir Matthew Gandy, « Cyborg Urbanization... », art. cité, pour une critique du politique de Bruno Latour.

${ }^{104}$ Notamment Ash Amin et Nigel Thrift : Arts of the Political, Durham/Londres, Duke University Press, 2013 et Seeing Like a City, Cambridge/Malden, Polity press, 2017.

105 « the city is everywhere and in everything ... They support unimaginably diverse social practices » : Ash Amin et Nigel Thrift, Cities: Reimagining the Urban, op. cit., p. 1-3 ; « the city....they remain extraordinarily complex entities - a mangle of machines, infrastructures, humans, non humans, institutions, networks,
} 
domination et les relations de pouvoir. En revanche ils considèrent comme caduque la réflexion centrée sur les acteurs ou les institutions. Pour eux, le pouvoir dans le monde urbain (l’État a disparu de leur monde) « est basé sur l'élan, plutôt que sur l'inscription. C'est le pouvoir en tant que force mobile et circulante qui, par la répétition constante des pratiques, produit des résultats similaires, moment par moment ${ }^{106} \gg$. Ils reprennent de manière très libre les concepts de diagramme de pouvoir et de gouvernementalité chez Foucault et Deleuze dans un feu d'artifice de figures de styles et de formulation qui s'enchaînent de manière plus virtuose qu'argumentée. Le politique urbain est vu comme constamment en mouvement, combinant imagination, délibération, interactions avec des étrangers, incertitudes, moments, par la création de publics contingents au gré des interactions au sein de l'assemblage machinique urbain. Les villes sont bien entendu ingouvernables et toute action collective plus ou moins liée à un gouvernement urbain est vouée à l'échec et sans intérêt. La démocratie est à réinventer pour deux raisons : premièrement, dans la perspective latourienne, les nonhumains doivent être intégrés dans toute délibération, et deuxièmement la démocratie représentative est en crise. C'est une vision du politique urbain où des assemblages évidemment complexes et toujours en mouvement dans une logique combinatoire et rhizomatique entrent ou non en interaction sans aucun principe de hiérarchie, en fonction de dynamiques internes incontrôlables produisant des moments, des directions non stabilisées, fétichisant l'incomplétude et quand même en lien, de manière mystérieuse, avec des éléments de gouvernements qui se doivent de voir la ville en terme de connaissance expérimentale de participation des citoyens, d'incertitude et d'absence de prévisibilité ${ }^{107}$. Cette ligne d'analyse est renforcée par les travaux sur les données produites dans les villes qui contribuent à réduire l'importance des réseaux physiques.

Antoine Picon insiste sur la conceptualisation de la ville en termes d'événements et d'occurrences combinant des éléments réels et virtuels où le politique résulte de l'affrontement classique entre des logiques de contrôles - par exemple par les données centralisées contre les initiatives citoyennes et horizontales qui innovent à partir des mêmes données $^{108}$. Pour cet auteur, le politique urbain est moins une affaire de rationalité que

metabolisms, matter and nature-where the coming together is itself constitutive of urbanity and its radiated effects » : ibid., p. 9.

${ }^{106}$ Le pouvoir dans le monde urbain « is based on momentum, rather than inscription. It is power as a mobile, circulating force which through the constant re-citation of practices, produces self-similar outcomes, moment by moment » : Ash Amin et Nigel Thrift, Cities: Reimagining the Urban, op. cit., p. 104 (notre traduction).

${ }^{107}$ Mariana Valverde, « Seeing Like a City: The Dialectic of Modern and Premodern Ways of Seeing in Urban Governance », Law \& Society Review, vol. 45, n² 2, 2011, p. 277-312.

${ }^{108}$ Antoine Picon, « Urban Infrastructure, Imagination and Politics: From The Networked Metropolis to the Smart City », International journal of urban and regional research, vol. 42, n 2, 2018, p. 263-275. 
d'imaginations dans le monde des possibles urbains.

Cette question des infrastructures définissant le politique urbain est également au cœur d'un courant d'anthropologie critique qui part des dispositifs matériels ${ }^{109}$. Akhil Gupta et ses collègues voient dans les infrastructures urbaines, des plus simples toilettes aux réseaux les plus sophistiqués un élément majeur de différenciation des modes de vie, de création d'inégalités, un enjeu majeur de redistribution. Pour eux, classiquement, la fourniture des grandes infrastructures urbaines était un processus peu politisé, garante d'une promesse de modernisme, de qualité de vie, de prospérité urbaine permettant à tous les citoyens de vaquer à leurs occupations, une version classique du libéralisme urbain. Les réseaux urbains étaient pensés en termes d'intégration urbaine. La concentration urbaine, les inégalités massives et la privatisation ont transformé ces infrastructures en outils de création d'inégalités entre quartiers d'une même ville. Le politique dans les villes est alors le politique de la vie quotidienne d'accès à l'eau, à l'énergie, à la mobilité ${ }^{110}$. Ces infrastructures représentent l'espoir d'une vie meilleure pour la population des quartiers pauvres, mais représentent les inégalités lorsque ces réseaux desservent d'abord les quartiers favorisés. Les anthropologues soulignent que l'accès à ces infrastructures est le meilleur marqueur d'accès à la citoyenneté ou à la construction du sujet politique à l'instar des aides sociales ${ }^{111}$. Dans la lignée de Dewey et Latour, ces travaux suggèrent que ces infrastructures, ces réseaux d'eau par exemple, participent de la production de leur public, d'une population urbaine constituée et gouvernée par ces réseaux, ou bien de l'exclusion de populations visiblement laissée de côté par les réseaux de transports, d'énergies, de traitement des déchets, de communication. La vie urbaine pour une partie de la population est rythmée par ces événements, ces coupures d'eau ou d'électricité, le ramassage aléatoire des poubelles. Les campagnes électorales se focalisent sur ces promesses de vie meilleure via un accès à un métro, ou à Internet.

Le débat autour de ces infrastructures urbaines est devenu un facteur majeur de politisation dans les grandes mégapoles. Dans les pays riches, la maintenance de ces réseaux est un énorme problème d'action collective (en témoigne le métro à New York qui court à la

\footnotetext{
${ }^{109}$ Nikhil Anand, Akhil Gupta, Hannah Appel (dir.), The Promise of Infrastructure, Durham/Londres, Duke University Press, 2018 ; Stephen Graham et Simon Marvin, Splintering Urbanism: Networked Infrastructures, Technological Mobilities and The Urban Condition, Londres, Routledge, 2001. Rowland Atkinson, Simon Parker, Emma Regina Morales, « Non-State Space: The Strategic Ejection of Dangerous and High Maintenance Urban Space », Territory, Politics, Governance, vol. 5, n 4, 2017, p. 443-458.

${ }^{110}$ Éric Verdeil, « Métropoles XXL: repenser le développement urbain à partir des réseaux d'infrastructure? », Métropolitiques, 26 octobre 2012, disponible en ligne (http://www.metropolitiques.eu/Metropoles-XXLrepenser-le.html).

${ }^{111}$ Voir par exemple Brian Larkin, « The politics and poetics of infrastructure », Annual Review of Anthropology, vol. 42, 2013, p. 327-343 ; Nikhil Anand, Hydraulic City: Water and the Infrastructures of Citizenship in Mumbai, Durham, Duke University Press, 2017.
} 
faillite) et provoque d'intenses débats.

\section{Conclusion}

La révolution industrielle en cours, la crise climatique, les migrations, le mouvement massif d'urbanisation mondialisé font des villes les grands laboratoires du monde du $21^{\mathrm{e}}$ siècle. Elles sont à la fois les leaders et les cibles.

À l'instar de Georg Simmel, on s'interroge aujourd'hui sur ce qui constitue l'urbanité contemporaine, ce que signifie être urbain ${ }^{112}$, sur les relations sociales et politiques qui s'inscrivent dans les villes, sur des rapports de pouvoir, des comportements, des modes d'exercice de l'autorité politique, sur les principes de justice ou la démocratie. Cet article a tenté de présenter les débats en cours sur le politique et la ville dans une perspective inscrite dans des dynamiques historiques privilégiant l'enchevêtrement des pouvoirs et des formes de désencastrement relatif des métropoles à l'égard des État, perspective qui ouvre un agenda de recherche sur les formes de gouvernementalisation des villes au sens de Michel Foucault, y compris en termes d'activités et de dispositifs matériels et de production des connaissances qui s'écartent un peu de la vulgate foucaldienne urbaine véhiculée par la géographie critique.

Poursuivre ces pistes de recherche sur ville, urbanisation et politique peut conduire à reprendre la discussion sur le républicanisme radical urbain, ou le municipalisme, dans un monde caractérisé par l'enchevêtrement des autorités politiques et des mobilisations à différentes échelles. Ces recherches incitent également à considérer les limites de la régulation politique et de la démocratie, peut être leur moindre importance dans les mondes urbains plus structurés par les technologies et les données, pour le meilleur comme pour le pire.

Enfin, ces recherches soulignent l'émergence d'un monde politique urbain de plus en plus connecté par des réseaux d'acteurs publics et privés où circulent des modèles, des imaginaires, des solutions, des formes de régulation politique. Au sein de ces métropoles grandes ou moyennes s'inventent des modèles politiques alternatifs, des modes de gestion des conflits, des formes de mobilisation en lien avec la crise climatique, des formes de participation politique, des manières d'intégrer des populations diverses, des imaginaires politiques. Si les tendances à la dénationalisation de l'autorité politique se confirment en Europe et ailleurs, il sera indispensable de renforcer la théorie politique des villes ${ }^{113}$.

\footnotetext{
${ }^{112}$ Sukriti Issar, « We Have Never Been Urban », Cities, 2018, p. 1-4. Michel Lussault, L'Homme spatial. La construction sociale de l'espace humain : La construction sociale de l'espace humain, Paris, Seuil, 2016.

${ }^{113}$ Je remercie Olivier Borraz, Gilles Pinson, Eduardo Marques, Côme Salvaire, Daniel Mouchard, ainsi que les réviseurs de la revue pour leurs commentaires précieux.
} 


\begin{abstract}
Auteur
Patrick Le Galès, FBA, MAE, est directeur de recherche au CNRS au Centre d'Etudes Européennes et de Politique Comparée de Sciences Po, Doyen de l'Ecole Urbaine de Sciences Po. Il a notamment publié : LE GALĖS (Patrick), Le Retour des villes européennes ?: sociétés urbaines, mondialisation, gouvernement et gouvernance, Paris, Presses de Sciences Po, 2010, ANDREOTTI (Alberta), LE GALÈS (Patrick), MORENO FUENTES (Javier Francesco), Un monde à la carte, les cadres supérieurs dans les villes européennes, Paris, PUF, 2016 KING (Desmond), LE GALES (Patrick), (eds.), Reconfigurating European states in crisis, Oxford, Oxford University Press , 2017; UGALDA (Vicente), LE GALÈS (Patrick), dir., Que se Gobierna ? El caso de la ciudad de Mexico, Mexico, Colejio de Mexico, 2018; COURMONT (Antoine), LE GALÈS (Patrick), (dir.), Ville et numérique, Paris, PUF, 2019; Le Galès, Patrick. "Neoliberalism and urban change: Stretching a good idea too far?." Territory, Politics, Governance 4.2 (2016): 154-172.
\end{abstract}

\begin{abstract}
Author
Patrick Le Galès, FBA, MAE, is CNRS research profesor at Sciences Po, Centre for European Studies and Comparative Politics, and Dean of Sciences Po Urban School. Publications include : LE GALÈS (Patrick), European Cities: Social Conflicts and Governance, Oxford, Oxford University Press, 2002; ANDREOTTI (Alberta), LE GALÈS (Patrick), MORENO FUENTES (Javier Francesco), , Globalizing minds, roots in the city, urban middle classes in European cities, Oxford, Blackwell/Wiley, 2015; KING (Desmond), LE GALES (Patrick), (eds.), Reconfigurating European states in crisis, Oxford, Oxford University Press , 2017; UGALDA (Vicente), LE GALÈS (Patrick), dir., Que se Gobierna ? El caso de la ciudad de Mexico, Mexico, Colejio de Mexico, 2018; COURMONT (Antoine), LE GALÈS (Patrick), (dir.), Ville et numérique, Paris, PUF, 2019; Le Galès, Patrick. "Neoliberalism and urban change: Stretching a good idea too far?." Territory, Politics, Governance 4.2 (2016): 154-172.
\end{abstract}

\title{
Résumé
}

\section{À la recherche du politique dans les villes}

Cet article est une contribution à la compréhension du politique dans les sociétés contemporaines plus ou moins mondialisées envisagé à partir des villes et non de l'État, en faisant l'hypothèse que les processus d'urbanisation et de mondialisation contemporains ouvrent des possibilités de différenciation du politique et des régulations politiques à l'échelle soit transnationale soit infranationale. Cette urbanisation planétaire remet en cause à l'inverse le politique identifié à un lieu et amène à un renouvellement des questions d'inégalités, de justice, de démocratie, de citoyenneté De manière différenciée de l'État, l'accumulation d'investissements matériels dans les villes en fait des concentrations originales de technologies qui peuvent s'analyser avec la sociologie de sciences comme des assemblages des systèmes socio-techniques où le politique se comprend en termes pragmatiques d'interactions quotidiennes. 


\begin{abstract}
Searching for politics in the urban world

This paper is a contribution to the understanding of politics in contemporary, globalising societies as seen from the perspective of cities rather than the state, on the assumption that contemporary urbanisation and globalisation processes open up possibilities for policy differentiation and policy regulation in cities. This global urbanisation challenges the politics identified with a place and leads to a renewal of questions of inequality, justice, democracy, citizenship, etc. Differently from the state, the accumulation of material investments in cities makes them original concentrations of technologies which can be analysed with the sociology of science as assemblages of socio-technical systems where politics is understood in pragmatic terms of daily interactions.
\end{abstract}

